Pacific

Journal of

Mathematics

CANONICAL CLASSES AND THE GEOGRAPHY OF NONMINIMAL LEFSCHETZ FIBRATIONS OVER $S^{2}$

YOSHIHISA SATO

Volume $262 \quad$ No. 1

March 2013 


\title{
CANONICAL CLASSES AND THE GEOGRAPHY OF NONMINIMAL LEFSCHETZ FIBRATIONS OVER $S^{2}$
}

\author{
YOSHIHISA SATO
}

\begin{abstract}
The Stipsicz conjecture on the fiber-sum decomposability of Lefschetz fibrations states that nonminimal Lefschetz fibrations over $S^{\mathbf{2}}$ are irreducible with respect to fiber-sum decompositions. We can conclude that such Lefschetz fibrations are prime and fundamental. In this paper, we determine the canonical classes of nonminimal Lefschetz fibrations admitting spheres of square -1 whose total intersection number with generic fiber is big. As a consequence, we consider the Kodaira dimension and the geography problem of such Lefschetz fibrations.
\end{abstract}

\section{Introduction}

If a 4-dimensional manifold $M$ admits some fibration structure, then we can understand its topology in detail. Elliptic surfaces, which are complex surfaces admitting elliptic fibrations whose generic fibers are smooth elliptic curves, were deeply studied by Kodaira, Kas, Moishezon and so on. Much is known about not only the topology of elliptic surfaces but also the differentiable structures on elliptic surfaces [Matsumoto 1986; Ue 1986; Donaldson 1987; Kametani and Sato 1994].

After that, symplectic structures are often studied as well as differentiable structures in 4-dimensional topology. In particular, Lefschetz fibrations have been studied in 4-dimensional symplectic topology since the latter half in the 1990's. A Lefschetz fibration is a smooth fibration of a smooth 4-manifold over a surface with finitely many critical points as complex analogues of Morse functions. Elliptic fibrations can be regarded as genus-1 Lefschetz fibrations. The importance of Lefschetz fibrations from the viewpoint of topology was reverified by Matsumoto [1996]. Lefschetz pencils and Lefschetz fibrations have played a major role in 4-dimensional symplectic topology by the support of the remarkable works of Donaldson [1998] and Gompf [1999], which imply that Lefschetz fibrations provide a topological

This research is supported by Grant-in-Aid for Scientific Research (C) (No. 23540096), Japan Society for the Promotion of Science.

MSC2010: primary 14J80, 57R17; secondary 14D06, 32Q65.

Keywords: Lefschetz fibrations, canonical class, geography, 4-manifolds, pseudoholomorphic curves,

Gromov invariants. 


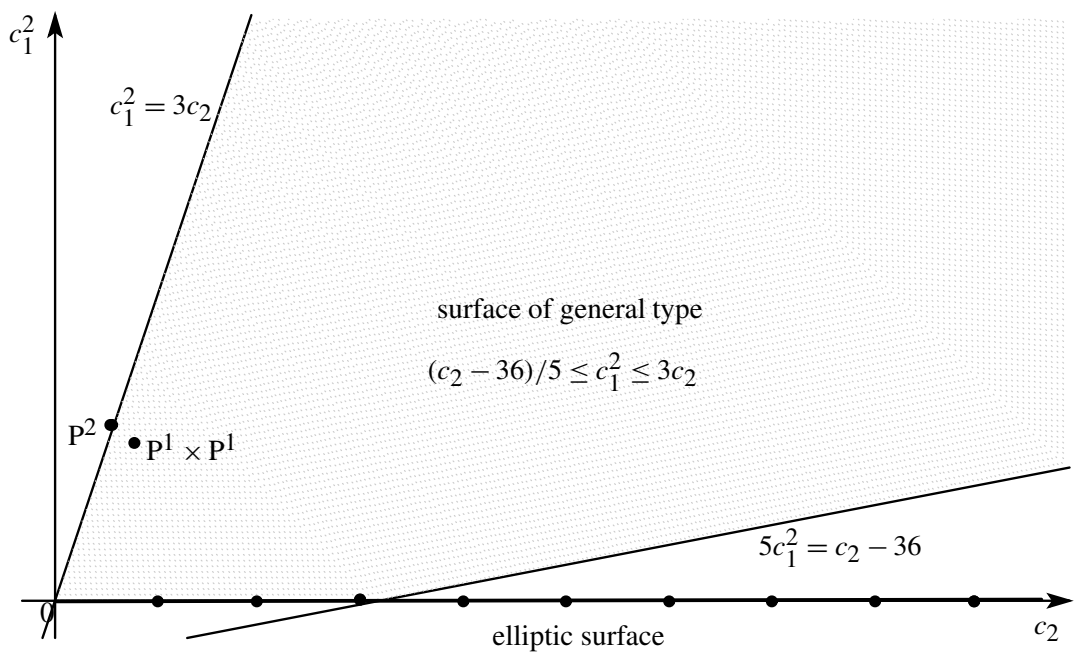

Figure 1. Geography of simply connected, minimal complex surfaces.

characterization of symplectic 4-manifolds and that most of symplectic 4-manifolds correspond to 4-manifolds with Lefschetz fibrations.

The geography problem in complex surfaces is the characterization of pairs of integers that are realized as the pairs $\left(c_{1}^{2}, c_{2}\right)$ of Chern numbers of complex surfaces, and it is well studied in algebraic geometry. By the classification of complex surfaces due to Kodaira, a simply connected complex surface is rational, elliptic or of general type. We know completely the range that rational surfaces and elliptic surfaces cover in the $\left(c_{1}^{2}, c_{2}\right)$-plane. Minimal surfaces of general type must satisfy $c_{1}^{2}, c_{2}>0$ (Noether inequality) and $\left(c_{2}-36\right) / 5 \leq c_{1}^{2} \leq 3 c_{2}$ (Bogomolov-MiyaokaYau inequality). In fact, the range of $\left(c_{1}^{2}, c_{2}\right)$ of simply connected, minimal complex surfaces is as shown in Figure 1.

A complex surface with even first Betti number $b_{1}$ is Kähler and so symplectic. Since any symplectic 4-manifold $M$ with symplectic structure $\omega$ admits an $\omega$-compatible almost-complex structure $J$, we can define Chern classes $c_{1}(T M, J), c_{2}(T M, J)$ for a symplectic 4-manifold $M$. Therefore, the geography for symplectic 4-manifolds comes into our mind. These problems are raised by McCarthy and Wolfson [1994]:

(1) Which pairs of integers are realized as $\left(c_{1}^{2}, c_{2}\right)$ of a symplectic 4-manifold?

(2) If there is a symplectic 4-manifold corresponding to a given lattice point $(m, n)$, how many symplectic 4 -manifolds realize $(m, n)$ as the pair $\left(c_{1}^{2}, c_{2}\right)$ of Chern numbers?

Questions (1) and (2) are called the geography problem and the botany problem, respectively. Since simply connected complex surfaces are symplectic, the range of 
$\left(c_{1}^{2}, c_{2}\right)$ for symplectic 4-manifolds contains the range for simply connected complex surfaces. The remarkable works of Donaldson and Gompf suggest that the geography of symplectic 4-manifolds is nearly the same as one of Lefschetz fibrations. Every lattice point $\left(c_{1}^{2}, c_{2}\right)$, except finitely many lying in $\left(c_{2}-36\right) / 5 \leq c_{1}^{2} \leq 2 c_{2}$, is realized as the total space of a Lefschetz fibration [Persson 1987]. On the other hand, Fintushel and Stern [1998] showed that there exists a minimal Lefschetz fibration that does not satisfy the Noether inequality. Stipsicz [1998] addressed the Bogomolov-Miyaoka-Yau inequality for Lefschetz fibrations. Therefore, there exists a difference between the range in the complex case and one in the symplectic case. See also [Gompf 1995; Stipsicz 1996].

Instead of investigating all of the objects, we restrict them to prime (or irreducible) things and examine these. Topologists often construct new manifolds by the cutand-paste method. As one can make a new manifold from given manifolds by taking the connected sum, we can make a new Lefschetz fibration from given Lefschetz fibrations by taking the fiber sum. In the category of Lefschetz fibrations, Lefschetz fibrations that cannot be decomposed as any nontrivial fiber sum are prime (or irreducible) with respect to fiber sum decompositions. Therefore, it is natural and enough to investigate the geography of irreducible Lefschetz fibrations, that is, Lefschetz fibrations that cannot be decomposed as any nontrivial fiber sum. Lefschetz fibrations with smooth spheres of square -1 have the following properties:

(1) Every projective complex surface admits the structure of a Lefschetz pencil and the notion of a Lefschetz pencil is important in the 4-dimensional topology. The blow-up of a Lefschetz pencil along the base locus yields a Lefschetz fibration with sections of square -1 . Conversely, by blowing down, we can obtain a Lefschetz pencil from a Lefschetz fibration with sections of square -1 .

(2) Any nonminimal Lefschetz fibration over $S^{2}$ that has smooth spheres of square -1 cannot be decomposed as a nontrivial fiber sum.

Fact (2) was conjectured by Stipsicz [2001]. The Stipsicz conjecture asserting the minimality of Lefschetz fibrations with fiber sum decomposability was proved by Usher [2006] affirmatively. In [Sato 2006] the author gave an independent and easier proof of the Stipsicz conjecture in the case of fiber genus 2. Thus, nonminimal Lefschetz fibrations $S^{2}$ are irreducible with respect to the fiber sum decompositions and we can conclude that such Lefschetz fibrations are fundamental.

The canonical class $K_{M}$ of a symplectic 4-manifold $(M, \omega, J)$ is defined by $K_{M}=-c_{1}(T M, J)$. Thus, if we determine the canonical class $K_{M}$, then we can calculate $c_{1}^{2}(T M, J)=K_{M}^{2}$. In this paper, we determine the canonical classes for nonminimal Lefschetz fibrations over $S^{2}$. By using $K_{X}^{2}$ of nonminimal Lefschetz fibrations $X \rightarrow S^{2}$, we can calculate the symplectic Kodaira dimension $\kappa^{s}$ and solve 
the geography problem for nonminimal Lefschetz fibrations. By using the symplectic Kodaira dimension $\kappa^{s}$, we can answer a question of Endo [2008, Problem 4.13] on the diffeomorphism type of three symplectic 4-manifolds admitting nonminimal Lefschetz fibrations.

Smith [2001b] showed the finiteness of the geography of genus-2 Lefschetz pencils; that is, there are only finitely many possible Chern pairs $\left(c_{1}^{2}, c_{2}\right)$ of genus-2 Lefschetz pencils. This implies that there an upper bound on the number of singular fibers of a genus-2 Lefschetz pencil. In fact, the number of singular fibers of such a pencil is less than or equal to 40. From this, the following question comes to our mind:

Question 1-1 (Smith [Auroux 2006b]). Is there an upper bound (in terms of the genus only) on the number of singular fibers of a Lefschetz fibration admitting a section of square -1 ?

In [Sato 2008], the author generalized Smith's result on genus-2 Lefschetz pencils to the geography on nonminimal genus-2 Lefschetz fibrations over $S^{2}$. In this paper, we consider the geography problem of nonminimal genus- $g(\geq 3)$ Lefschetz fibrations over $S^{2}$ and show the finiteness of the geography of certain classes of nonminimal genus- $g$ Lefschetz fibrations, which gives us a partial answer for Question 1-1. For example, in the case where nonminimal Lefschetz fibrations are hyperelliptic and have only $(-1)$-sections as smooth spheres of square -1 , we have:

Theorem 1-2. For $g \geq 3$, there are only finitely many possible Chern pairs $\left(c_{1}^{2}, c_{2}\right)$ of hyperelliptic genus- $g$ Lefschetz fibrations with $2 g-2$ or $2 g-3$ sections of square -1 whose total spaces are neither the blow-up of a rational surface nor the blow-up of a ruled surface. As a consequence, there is an upper bound on the number of singular fibers of such a Lefschetz fibration. In fact, for any such hyperelliptic genus-g Lefschetz fibration $f: X \rightarrow S^{2}$, the number $\mu(f)$ of singular fibers of $f$ satisfies

$$
\mu(f) \leq \frac{(8 g-9)(2 g+1)}{g-1}+\sum_{h=1}^{[g / 2]} \frac{16 g^{2}-11 g-8}{12 h(g-h)-(2 g+1)} .
$$

We can answer Question 1-1 in a generic situation; see Section 6. On the other hand, considering the fiber sum construction, we see that minimal Lefschetz fibrations can have arbitrarily many singular fibers.

The organization of this paper is as follows: In Sections 2-3, we recall the notion of Lefschetz fibrations over $S^{2}$ and give some examples of nonminimal Lefschetz fibrations. In Section 4, we consider the geography of symplectic 4-manifolds and Lefschetz fibrations. In Section 5, we determine the canonical classes of nonminimal Lefschetz fibrations and answer Endo's question. In Section 6, we show 
the finiteness of the geography of nonminimal hyperelliptic Lefschetz fibrations. Furthermore, we consider the geography of nonminimal, nonhyperelliptic genus-3 Lefschetz fibrations.

\section{Lefschetz fibrations over $S^{2}$}

The definition of Lefschetz fibrations. A smooth map $f: X \rightarrow \Sigma$ from a closed, connected, oriented smooth 4-manifold $X$ onto a closed, connected, oriented smooth 2-manifold $\Sigma$ is said to be a Lefschetz fibration if $f$ admits finitely many critical points $C=\left\{p_{1}, p_{2}, \ldots, p_{k}\right\}$ on which $f$ is injective and around which there are orientation-preserving complex coordinate neighborhoods such that locally $f$ can be expressed as $f\left(z_{1}, z_{2}\right)=z_{1}^{2}+z_{2}^{2}$. It is a consequence of this definition that $\left.f\right|_{X \backslash C}: X \backslash C \rightarrow \Sigma \backslash f(C)$ is a smooth fiber bundle with fiber a closed oriented 2-manifold.

If a generic fiber that is the inverse image of a regular value has genus $g$, or equivalently if $\left.f\right|_{X \backslash C}$ is a surface bundle with fiber a closed orientable surface of genus $g$, we refer to $f$ as a genus- $g$ Lefschetz fibration. Moreover, we assume that $f$ is relatively minimal, that is, there is no fiber containing a sphere of square -1 . Two Lefschetz fibrations $f: X \rightarrow \Sigma$ and $f^{\prime}: X^{\prime} \rightarrow \Sigma^{\prime}$ are isomorphic if there are diffeomorphisms $\Phi: X \rightarrow X^{\prime}$ and $\varphi: \Sigma \rightarrow \Sigma^{\prime}$ such that $f^{\prime} \circ \Phi=\varphi \circ f$. In this paper, we will assume that a base space $\Sigma$ is a 2-sphere.

A fiber containing a critical point is called a singular fiber, which is obtained by collapsing a simple closed curve, called a vanishing cycle, on a nearby generic fiber to a point. A singular fiber is called reducible or irreducible according to whether the corresponding vanishing cycle separates or dose not separate in the generic fiber. In particular, if a vanishing cycle $\alpha$ separates the closed surface $\Sigma_{g}$ of genus $g$ into two components with genera $h$ and $g-h(1 \leq h \leq[g / 2])$, then the reducible singular fiber corresponding to $\alpha$ is said to be of type $I I_{h}$.

Let $\Gamma_{g}$ be the mapping class group of genus $g$, namely the group of all isotopy classes of orientation-preserving self-diffeomorphisms of $\Sigma_{g}$. The local monodromy around a singular fiber of a Lefschetz fibration $f: X \rightarrow S^{2}$ is a positive Dehn twist $\tau_{a}$ along the corresponding vanishing cycle $a$. See Figure 2. Since the base space of $f$ is a 2-sphere, the product of all the local monodromies of $f$ is trivial in $\Gamma_{g}$. Such a relation in $\Gamma_{g}$

$$
t_{a_{1}} t_{a_{2}} \cdots t_{a_{\mu}}=1
$$

is called a positive relation, where $a_{1}, a_{2}, \ldots, a_{\mu}$ are vanishing cycles of $f$ and each $t_{a_{i}}$ is the isotopy class of $\tau_{a_{i}}$ in $\Gamma_{g}$.

Isomorphism classes of Lefschetz fibrations are determined by the monodromy representations as follows: 


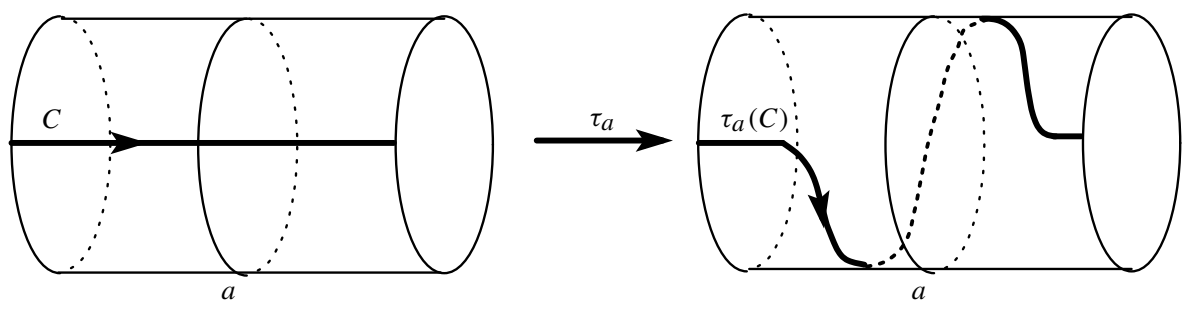

Figure 2. A positive Dehn twist.

Theorem 2-1 [Matsumoto 1996]. Suppose that $g \geq 2$. Then, there is a one-to-one correspondence

$$
\left\{\begin{array}{l}
\text { isomorphism classes of Lefschet } \\
\text { fibrations with } n \text { singular fibers }
\end{array}\right\} \longleftrightarrow\{\text { conjugacy classes of } \rho\},
$$

where $\rho: \pi_{1}\left(S^{2} \backslash \bigcup f\left(p_{i}\right), b_{0}\right) \rightarrow \Gamma_{g}$ is the monodromy representation.

From this theorem, it is well-known that a genus- $g$ Lefschetz fibration is characterized by a positive relation $t_{a_{1}} t_{a_{2}} \cdots t_{a_{\mu}}=1$ in $\Gamma_{g}$ up to Hurwitz equivalence and simultaneous conjugation of all factors by a same element in $\Gamma_{g}$.

A Lefschetz pencil is a nonempty finite set $B=\left\{b_{1}, b_{2}, \ldots, b_{\ell}\right\}$ of $X$, called the base locus, together with a smooth map $f: X \backslash B \rightarrow \mathbb{C} \mathrm{P}^{1}$ such that each $b_{i}$ has an orientation-preserving complex coordinate neighborhood in which locally $f$ can be expressed as $f\left(z_{1}, z_{2}\right)=z_{1} / z_{2}$, and each critical point of $f$ has a local coordinate neighborhood as a Lefschetz fibration. By the definitions of Lefschetz fibrations and pencils, the blow-up at the base locus points of a Lefschetz pencil yields a Lefschetz fibration over $S^{2}$ with sections of square -1 . It is well-known that every projective complex surface admits a Lefschetz pencil, which is generalized to symplectic 4-manifolds as follows:

Theorem 2-2 [Donaldson 1998]. Every symplectic 4-manifold admits a Lefschetz pencil whose closed fibers are symplectic submanifolds.

A result of Thurston [1976] on symplectic structures of surface-bundles over closed oriented surfaces can be generalized as follows to 4-manifolds admitting Lefschetz fibrations.

Theorem 2-3 [Gompf and Stipsicz 1999]. Let $f: X \rightarrow S^{2}$ be a Lefschetz fibration and $[F]$ denote the homology class of the fiber. If $[F] \neq 0$ in $H_{2}(X ; \mathbb{R})$, then $X$ admits a symplectic structure such that fibers are symplectic submanifolds.

If the fiber genus $g$ is greater than 1, then the homology class of a generic fiber of $f$ is not torsion in $H_{2}(X ; \mathbb{Z})$, and so this theorem states that such an $X$ admits a symplectic structure such that fibers of $f$ are symplectic submanifolds.

From now on, we suppose that the fiber genus $g$ is greater than 1 and we can use the symplectic topology. Then, combining the remarkable theorems of 
Donaldson and Gompf gives the following topological characterization of symplectic 4-manifolds.

Corollary 2-4. A 4-manifold $X$ admits a symplectic structure if and only if it admits a Lefschetz pencil.

Proof. By Theorem 2-2, a symplectic 4-manifold $X$ admits a Lefschetz pencil. If $X$ admits a Lefschetz pencil, then the blow-up at the base locus points of a Lefschetz pencil yields a Lefschetz fibration $f: X \# n \overline{\mathbb{C P}^{2}} \rightarrow S^{2}$ with sections of square -1 . Let $F$ be a generic fiber of $f$ and $E$ a $(-1)$-section of $f$. Since $F \cdot E=1$, the homology class of $F$ is nontrivial in $H_{2}(X \# n \overline{\mathbb{C P}} ; \mathbb{R})$, and so it follows from Theorem 2-3 that $X \# n \overline{\mathbb{C P}^{2}}$ admits a symplectic structure $\omega$. If $X$ is rational or ruled, then $X$ has a symplectic structure. Suppose that $X$ is neither rational nor ruled. Then, by the (-1)-curve theorem [Li and Liu 1995; Taubes 1995; 1996], we regard a smooth $(-1)$-section as an $\omega$-symplectic sphere of square -1 . Hence, the symplectic blow-down of $X \# n \overline{\mathbb{C P}} \overline{\mathrm{P}}^{2}$ yields a symplectic structure on $X$.

Let $f_{i}: X_{i} \rightarrow S^{2}(i=1,2)$ be a genus- $g$ Lefschetz fibration. Removing regular neighborhoods $N\left(F_{1}\right), N\left(F_{2}\right)$ of generic fibers $F_{1}, F_{2}$ in each, we glue these open remainders along their boundaries by using a fiber-preserving diffeomorphism $\varphi: \partial\left(X_{1}-\operatorname{Int} N\left(F_{1}\right)\right) \rightarrow \partial\left(X_{2}-\operatorname{Int} N\left(F_{2}\right)\right)$ with $f_{2} \circ \varphi=f_{1}$ on $\partial\left(X_{1}-\operatorname{Int} N\left(F_{1}\right)\right)$. We denote the resulting 4-manifold by $X_{1} \#_{F} X_{2}$, that is, $X_{1} \#_{F} X_{2}=\left(X_{1}-\right.$ Int $\left.N\left(F_{1}\right)\right) \cup_{\varphi}\left(X_{2}-\operatorname{Int} N\left(F_{2}\right)\right)$. Then $X_{1} \#_{F} X_{2}$ admits a genus- $g$ Lefschetz fibration $f_{1} \#_{F} f_{2}: X_{1} \#_{F} X_{2} \rightarrow S^{2}$ associated to $f_{1}$ and $f_{2}$. We call the genus- $g$ Lefschetz fibration $f_{1} \#_{F} f_{2}: X_{1} \#_{F} X_{2} \rightarrow S^{2}$ the fiber sum of $f_{1}$ and $f_{2}$. The diffeomorphism type of $X_{1} \#_{F} X_{2}$ might depend on the choice of the gluing diffeomorphism $\varphi$. In fact, Ozbagci and Stipsicz [2000] constructed infinitely many Lefschetz fibrations as the fiber sums from the same building blocks by using various gluing diffeomorphisms. However, for the sake of brevity, we do not record those dependencies. By taking the fiber sums, we can obtain infinitely many genus- $g$ Lefschetz fibrations. On the other hand, Stipsicz [2001] and Smith [2001a] showed that, if a Lefschetz fibration has a $(-1)$-section, then it cannot be decomposed as any nontrivial fiber sum. Furthermore, Usher [2006] showed that no nonminimal Lefschetz fibration can be decomposed as a nontrivial fiber sum (the Stipsicz conjecture).

Therefore, nonminimal Lefschetz fibrations are "irreducible" building blocks in the fiber sum construction. Thus, we consider nonminimal Lefschetz fibrations in this paper.

The signature of Lefschetz fibrations. The Hirzebruch signature theorem implies that the pair $\left(c_{1}^{2}, c_{2}\right)$ of Chern numbers is determined by the signature and the Euler characteristic. So, when we consider the geography of Lefschetz fibrations later, it is important to calculate the signature and the Euler characteristic of a 4-manifold 


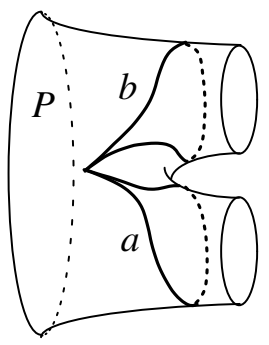

Figure 3. A pair of pants.

admitting a Lefschetz fibration. Every singular fiber of a genus- $g$ Lefschetz fibration $f: X \rightarrow S^{2}$ contributes +1 to the Euler characteristic $e(X)$. If the fibration $f$ has $\mu$ singular fibers, then we have $e(X)=4(1-g)+\mu$.

Compared with the calculation of the Euler characteristic, it is difficult to calculate the signature of $X$. Now we introduce two signature formulae. One is the Matsumoto-Endo formula for hyperelliptic Lefschetz fibrations and the other is the Smith formula for general (possibly nonhyperelliptic) Lefschetz fibrations. Let $F_{1}, F_{2}, \ldots, F_{\mu}$ be singular fibers of $f: X \rightarrow S^{2}$. Let $N\left(F_{i}\right)$ denote the tubular neighborhood of $F_{i}(i=1,2, \ldots, \mu)$. We set $X_{0}=X-\bigcup_{i=1}^{\mu} N\left(F_{i}\right)$. Then the restriction $f_{\mid X_{0}}: X_{0} \rightarrow f\left(X_{0}\right)$ is the associated $\Sigma_{g}$-bundle over the punctured sphere. Since an irreducible singular fiber and a reducible singular fiber contribute 0 and -1 to the signature $\sigma(X)$, respectively, it follows from the Novikov additivity that we have

$$
\sigma(X)=\sigma\left(X_{0}\right)-\sum_{h=1}^{[g / 2]} s_{h},
$$

where $s_{h}$ denotes the number of singular fibers of type $I I_{h}$. The signature $\sigma\left(X_{0}\right)$ of the bundle part $X_{0}$ can be calculated from the signature cocycle $\tau_{g}$, which is a 2-cocycle of the Siegel modular group $\operatorname{Sp}(2 g ; \mathbb{Z})$ [Meyer 1973]. Let $P=$ $S^{2}-\bigsqcup_{i=1}^{3}$ Int $D_{i}^{2}$ be a pair of pants and $E(\alpha, \beta) \rightarrow P$ the $\Sigma_{g}$-bundle defined by monodromies $\alpha, \beta \in \Gamma_{g}$.

Then, Meyer [1973] showed that for the signature of $E(\alpha, \beta)$ we have

$$
\sigma(E(\alpha, \beta))=-\tau_{g}(\alpha, \beta) .
$$

Since $f$ has $\mu$ singular fibers, we can decompose the $\mu$-punctured sphere $f\left(X_{0}\right)$ into $\mu-2$ pairs $P_{1}, P_{2}, \ldots, P_{\mu-2}$ of pants as in Figure 4 .

Then it follows from Novikov additivity and Meyer's theorem that we have

$$
\sigma\left(X_{0}\right)=\sum_{i=1}^{\mu-2} \sigma\left(f^{-1}\left(P_{i}\right)\right)=-\sum_{i=1}^{\mu} \tau_{g}\left(t_{a_{i-1}} \cdots t_{a_{2}} t_{a_{1}}, t_{a_{i}}\right) .
$$




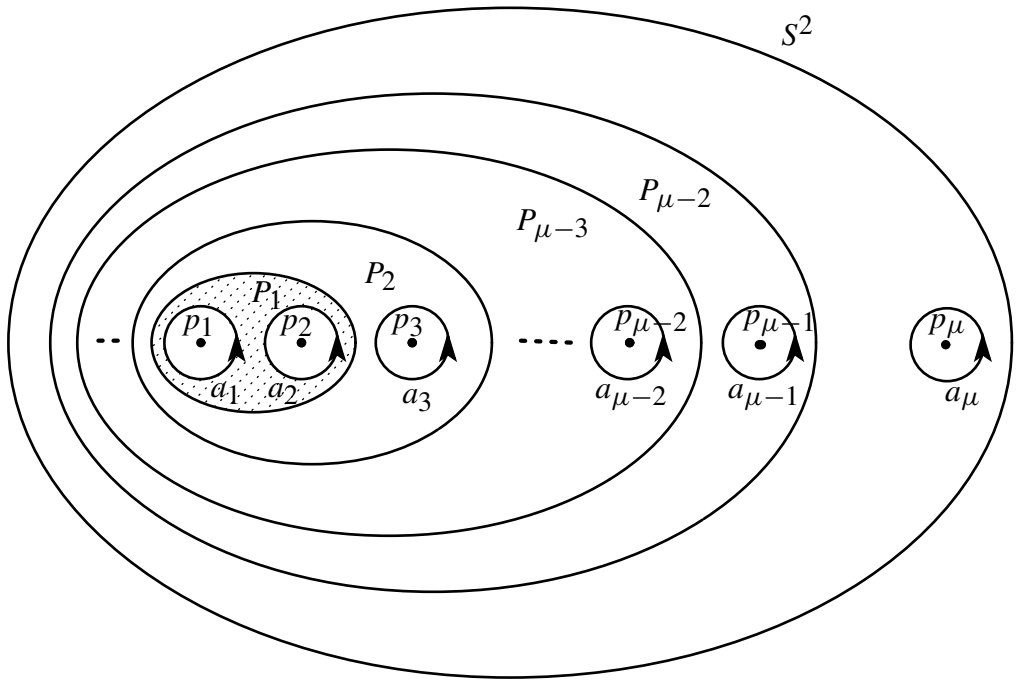

Figure 4. Decomposition of $f\left(X_{0}\right)$ into $\mu-2$ pairs of pants.

Here $a_{1}, a_{2}, \ldots, a_{\mu}$ are vanishing cycles of $f$ and $t_{a_{0}}$ denotes the identity map. Thus, in terms of the signature cocycle $\tau_{g}$ and monodromies $t_{a_{1}}, t_{a_{2}}, \ldots, t_{a_{\mu}}$, the signature of $X$ is given by

$$
\sigma(X)=-\sum_{i=1}^{\mu} \tau_{g}\left(t_{a_{i-1}} \cdots t_{a_{2}} t_{a_{1}}, t_{a_{i}}\right)-\sum_{h=1}^{[g / 2]} s_{h} .
$$

The Matsumoto-Endo signature formula. A hyperelliptic Lefschetz fibration is a Lefschetz fibration whose monodromy representation $\rho$ is equivalent to one taking isotopy classes commuting with the hyperelliptic involution $\iota: \Sigma_{g} \rightarrow \Sigma_{g}$. Since the hyperelliptic mapping class group $\Gamma_{2}^{\text {hyp }}$ of genus 2 agrees with $\Gamma_{2}$, every genus-2 Lefschetz fibration is hyperelliptic.

When we restrict the signature cocycle $\tau_{g}$ to the hyperelliptic mapping class group $\Gamma_{g}^{\text {hyp }}$, its cohomology class $\left[\tau_{g}^{H}\right] \in H^{2}\left(\Gamma_{g}^{\text {hyp }} ; \mathbb{Z}\right)$ is of finite order. So we can calculate the terms of signature cocycles by the coboundary maps called Meyer's functions. Matsumoto [1996] and Endo [2000] calculated Meyer's functions and obtained the signature formula for hyperelliptic Lefschetz fibrations.

Theorem 2-5 [Matsumoto 1996; Endo 2000]. Suppose that $f: X \rightarrow S^{2}$ is a genus- $g$ hyperelliptic Lefschetz fibration with $n_{0}$ irreducible singular fibers and $s_{h}$ singular fibers of type $I I_{h}(h=1,2, \ldots,[g / 2])$. Then, we have

$$
\sigma(X)=-\frac{g+1}{2 g+1} n_{0}+\sum_{h=1}^{[g / 2]}\left(\frac{4 h(g-h)}{2 g+1}-1\right) s_{h} .
$$


Smith's signature formula. Smith obtained the signature formula for (possibly nonhyperelliptic) Lefschetz fibrations by using the geometry of the moduli space of stable curves. We denote the Deligne-Mumford compactified moduli space of stable curves of genus $g$ by $\overline{\mathcal{M}}_{g}$. Let $f: X \rightarrow S^{2}$ be a genus- $g$ Lefschetz fibration. Then, we can have a symplectic structure on $X$ such that each fiber $f^{-1}(x)$ is a pseudoholomorphic curve. Since a 2-dimensional almost-complex structure is integrable, each fiber $f^{-1}(x)$ determines a point in the Deligne-Mumford compactified moduli space $\bar{M}_{g}$.

Thus we can define the moduli map $\phi_{f}: S^{2} \rightarrow \bar{M}_{g}$ of $f$ by

$$
\phi_{f}(x):=\left[f^{-1}(x)\right] \in \bar{M}_{g} \text { for all } x \in S^{2} .
$$

In particular, if $f: X \rightarrow \mathbb{C P}$ is holomorphic, then the image $\phi_{f}\left(\mathbb{C P}^{1}\right)$ is a rational curve in $\bar{M}_{g}$.

Theorem 2-6 [Smith 1999]. For any genus-g Lefschetz fibration $f: X \rightarrow S^{2}$ with $\mu$ singular fibers, namely $\mu=n_{0}+\sum_{h=1}^{[g / 2]} s_{h}$, the signature of $X$ is given by

$$
\sigma(X)=4\left\langle c_{1}(\lambda),\left[\phi_{f}\left(S^{2}\right)\right]\right\rangle-\mu,
$$

where $\lambda \rightarrow \bar{M}_{g}$ denotes the Hodge bundle with fiber $\wedge^{g} H^{0}\left(C ; K_{C}\right)$, the determinant line above $[C]$.

For a projective fibration $f: X \rightarrow \mathbb{C} \mathrm{P}^{1}$, this theorem follows from Mumford's formula. Smith's formula is a generalization of Atiyah's formula for smooth fibrations, and related work by Meyer.

\section{Examples of Lefschetz fibrations}

Let $\Gamma_{g}$ be the mapping class group of $\Sigma_{g}$. For elements $\varphi, \psi \in \Gamma_{g}$, the product $\psi \cdot \varphi($ or $\psi \varphi$ ) stands for applying $\varphi$ first and then $\psi$.

Let $c_{1}, c_{2}, \ldots, c_{2 g+1}$ be the curves on $\Sigma_{g}$ illustrated in Figure 5. The isotopy classes of the positive Dehn twists $\tau_{c_{1}}, \tau_{c_{2}}, \ldots, \tau_{c_{2 g+1}}$ along $c_{1}, c_{2}, \ldots, c_{2 g+1}$ are

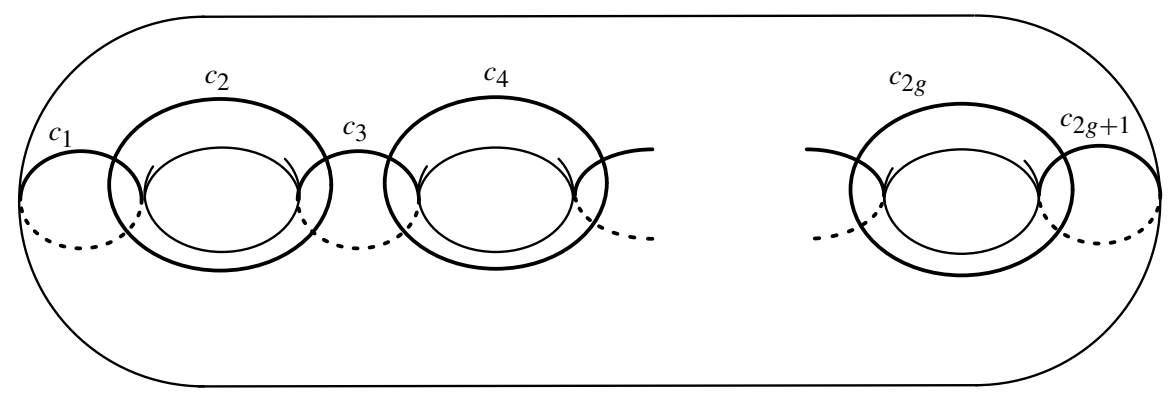

Figure 5. Lickorish generators. 
Lickorish generators of the mapping class group $\Gamma_{g}$ of genus $g$. For the sake of brevity, we denote the isotopy class of the positive Dehn twist $\tau_{a}$ along the curve $a$ by the same symbol $a$.

It is well-known that $\Gamma_{g}$ has the following positive relations:

$$
\begin{aligned}
& W_{1}:\left(c_{1} \cdot c_{2} \cdots c_{2 g} \cdot c_{2 g+1} \cdot c_{2 g+1} \cdot c_{2 g} \cdots c_{2} \cdot c_{1}\right)^{2}=1, \\
& W_{2}:\left(c_{1} \cdot c_{2} \cdots c_{2 g} \cdot c_{2 g+1}\right)^{2 g+2}=1, \\
& W_{3}:\left(c_{1} \cdot c_{2} \cdots c_{2 g}\right)^{4 g+2}=1 .
\end{aligned}
$$

From these positive relations, we can construct hyperelliptic genus- $g$ Lefschetz fibrations with only irreducible singular fibers and with sections of square -1 . Furthermore, these Lefschetz fibrations are double branched covers of the Hirzebruch surfaces and so holomorphic. The total space of the Lefschetz fibration corresponding to $W_{1}$ is diffeomorphic to $\mathbb{C P}^{2} \#(4 g+5) \overline{\mathbb{C P}}{ }^{2}$.

Examples of nonminimal genus-2 Lefschetz fibrations. The Hirzebruch surface $\mathbb{F}_{n}=\mathbb{P}\left(\mathcal{O}_{\mathbb{C P}^{1}} \oplus \mathcal{O}_{\mathbb{C P}^{1}}(n)\right)$ has two disjoint holomorphic sections $\Delta_{n}$ and $\Delta_{-n}$ of square $\pm n$.

(1) $M_{1}=\mathbb{C P}^{2} \# 13 \overline{\mathbb{C P}}{ }^{2}:$ The positive relation $W_{1}:\left(c_{1} \cdot c_{2} \cdot c_{3} \cdot c_{4} \cdot c_{5}^{2} \cdot c_{4} \cdot c_{3} \cdot c_{2} \cdot c_{1}\right)^{2}=1$ describes the genus-2 Lefschetz fibration on the rational surface $M_{1}$ obtained as a double covering of $\mathbb{F}_{0}$ branched along a smooth algebraic curve in the linear system $|6 \Delta+2 F|$. This fibration is obtained from the composition of the covering projection with the bundle projection $\mathbb{F}_{0} \rightarrow S^{2}$ and has 20 irreducible singular fibers and sections of square -1 .

(2) $M_{2}=K 3 \# 2 \overline{\mathbb{C P}^{2}}:$ The positive relation $W_{2}:\left(c_{1} \cdot c_{2} \cdot c_{3} \cdot c_{4} \cdot c_{5}\right)^{6}=1$ describes the genus-2 Lefschetz fibration on $M_{2}$ obtained as a double covering of $\mathbb{F}_{1}=\mathbb{C P}^{2} \# \overline{\mathbb{C P}}$ branched along a smooth algebraic curve in the linear system $|6 L|$, where $L$ is a line in $\mathbb{C P}^{2}$ avoiding the blown-up point. This fibration has 30 irreducible singular fibers and sections of square -1 .

(3) $M_{3}=H^{\prime}(1)$ (Horikawa surface) : The positive relation $W_{3}:\left(c_{1} \cdot c_{2} \cdot c_{3} \cdot c_{4}\right)^{10}=1$ describes the genus-2 Lefschetz fibration on $M_{3}$ obtained as a double covering of $\mathbb{F}_{2}$ branched along the disjoint union of a smooth curve in the linear system $\left|5 \Delta_{2}\right|$ and $\Delta_{-2}$. This fibration has 40 irreducible singular fibers and a section of square -1 . This section is a lift of the component of the branched set coming from $\Delta_{-2}$. On the other hand, a fiber sum of two copies of the rational genus-2 Lefschetz fibration $\mathbb{C P}^{2} \# 13 \overline{\mathbb{C P}} \rightarrow S^{2}$ is a genus-2 Lefschetz fibration, which has 40 irreducible singular fibers and the total space is homeomorphic to $H^{\prime}(1)$ but not diffeomorphic. 
(4) $S^{2} \times T^{2} \# 4 \overline{\mathbb{C P} 2}$ : Matsumoto showed that $S^{2} \times T^{2} \# 4 \overline{\mathbb{C P}^{2}}$ has a genus-2 Lefschetz fibration with 6 irreducible singular fibers and 2 reducible singular fibers. This also has a section of square -1 . The positive relation describing this fibration is $\left(\alpha_{1} \cdot \sigma \cdot \alpha_{2} \cdot \alpha_{3}\right)^{2}=1$, where $\alpha_{1}, \alpha_{2}, \alpha_{3}$ and $\sigma$ are given by positive Dehn twists along the curves indicated in Figure 6.
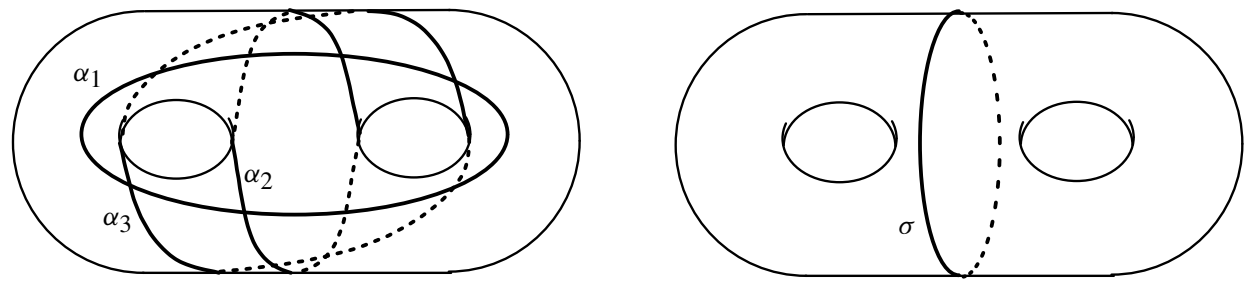

Figure 6

(5) Auroux's genus-2 Lefschetz fibration: Auroux [2003] constructed the interesting genus-2 Lefschetz fibration $f: X \rightarrow \mathbb{C P}^{1}$ with 28 irreducible singular fibers and one reducible singular fiber. This fibration is nonminimal but does not admit section of square (-1). See [Sato 2008]. The positive relation corresponding to it is given as follows:

$\sigma \cdot\left(c_{3} \cdot c_{4} \cdot c_{5} \cdot c_{2} \cdot c_{3} \cdot c_{4} \cdot c_{1} \cdot c_{2} \cdot c_{3}\right)^{2} \cdot\left(c_{1} \cdot c_{2} \cdot c_{3} \cdot c_{4} \cdot c_{5} \cdot c_{5} \cdot c_{4} \cdot c_{3} \cdot c_{2} \cdot c_{1}\right)=1$.

For other examples of nonminimal genus-2 Lefschetz fibrations, see [Sato 2008].

\section{Examples of nonminimal genus-3 Lefschetz fibrations.}

(1) $M_{1}, M_{2}$ and $M_{3}$ corresponding to positive relations $W_{1}, W_{2}$ and $W_{3}$ for $g=3$ have nonminimal, hyperelliptic and holomorphic genus-3 Lefschetz fibrations.

(2) $S^{2} \times T^{2} \# 8 \overline{\mathbb{C P}^{2}}$ : This has a nonhyperelliptic genus-3 Lefschetz fibration with positive relation $\left(\alpha_{1} \cdot \alpha_{2} \cdot \alpha_{3} \cdot \alpha_{4} \cdot \beta_{1}^{2} \cdot \beta_{2}^{2}\right)^{2}$ indicated in Figure 7. This fibration also has a section of square -1 .

(3) Fuller's example : Fuller constructed a nonhyperelliptic and nonholomorphic genus-3 Lefschetz fibration with positive relation

$$
\left(\beta_{1} \cdot \beta_{2} \cdot c_{4} \cdot c_{3} \cdot c_{2} \cdot c_{1} \cdot c_{5} \cdot c_{4} \cdot c_{3} \cdot c_{2} \cdot c_{6} \cdot c_{5} \cdot c_{4} \cdot c_{3} \cdot\left(c_{1} \cdots c_{6}\right)^{10}\right)=1,
$$
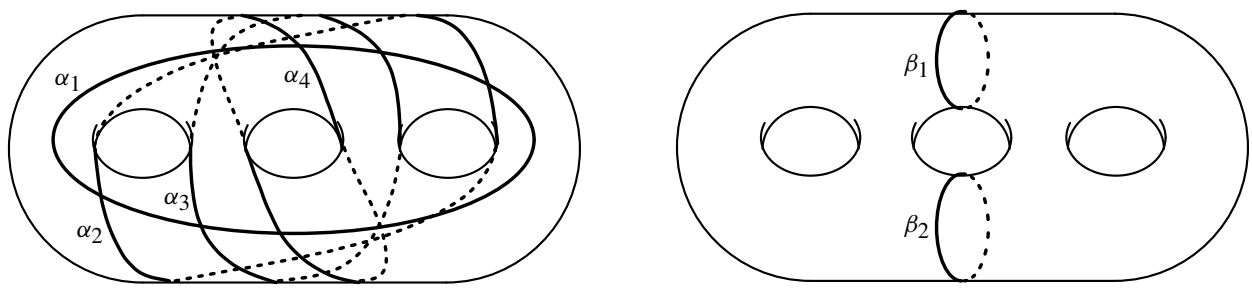

Figure 7 
where $\beta_{1}$ and $\beta_{2}$ are given by positive Dehn twists along curves indicated in Figure 7. This fibration also has a section of square -1 .

(4) $T^{4} \# 4 \overline{\mathbb{C P}^{2}}$ : Smith [2001c] showed that $T^{4} \# 4 \overline{\mathbb{C P}^{2}}$ has a hyperelliptic and holomorphic genus-3 Lefschetz fibration with 12 irreducible singular fibers and 4 sections of square -1 . This fibration is obtained by using the inverse of the usual Kummer construction of a $K 3$ surface which is elliptically fibered over $S^{2}$ with 16 disjoint (-2)-spheres containing 4 sections and 12 singular fibers.

(5) Fermat surface of degree 4 : The Fermat surface of degree 4 is the smooth hypersurface in $\mathbb{C} P^{3}$ defined by the equation $z_{0}^{4}+z_{1}^{4}+z_{2}^{4}+z_{3}^{4}=0$. Kuno [2010] proved that this surface admits a genus-3 Lefschetz pencil with 4 base locus points. The blow-up of this surface at the base locus points yields a nonminimal, nonhyperelliptic, holomorphic genus-3 Lefschetz fibration with only 36 irreducible singular fibers and 4 sections of square -1 . See [Kuno 2010] for its monodromies.

Examples of nonminimal genus-g Lefschetzfibrations. Endo [2008] generalized some parts of Chakiris' construction of holomorphic genus-2 Lefschetz fibrations topologically to give many examples of nonminimal hyperelliptic Lefschetz fibrations of arbitrary genus. Their examples are given in terms of positive relations in mapping class groups. Now, from Endo's list, we introduce some examples that we investigate in Section 5.

For simple closed curves $c, a_{1}, a_{2}, \ldots, a_{r}$ on $\Sigma_{g}$ and

$$
W=a_{r}^{\varepsilon_{r}} \cdots a_{2}^{\varepsilon_{2}} a_{1}^{\varepsilon_{1}} \quad\left(\varepsilon_{1}, \varepsilon_{2}, \ldots, \varepsilon_{r} \in\{ \pm 1\}\right),
$$

we put ${ }_{W}(c):=\tau_{a_{r}}^{\varepsilon_{r}} \cdots \tau_{a_{2}}^{\varepsilon_{2}} \tau_{a_{1}}^{\varepsilon_{1}}(c)$. Furthermore, for a factorization $V=c_{i_{1}} c_{i_{2}} \cdots c_{i_{r}}$, we put ${ }_{W} V:={ }_{W}\left(c_{i_{1}}\right)_{W}\left(c_{i_{2}}\right) \cdots_{W}\left(c_{i_{r}}\right)$.

We define words $I, J, C_{\mathrm{I}}, C_{\mathrm{II}}, P, Q$ and $R$ in $\Gamma_{g}^{\text {hyp }}$ as follows. Here, $d$ denotes the boundary curve of a regular neighborhood of $c_{1} \cup c_{2} \cup \cdots \cup c_{2[g / 2]}$.

$$
\begin{aligned}
I & :=c_{1} \cdot c_{2} \cdots c_{2 g} \cdot c_{2 g+1}^{2} \cdot c_{2 g} \cdots c_{2} \cdot c_{1}, \\
J & :=\left(c_{1} \cdot c_{2} \cdots c_{2 g}\right)^{2 g+1}, \\
C_{\mathrm{I}} & :=\left(c_{1} \cdot c_{2} \cdots c_{2 g+1}\right)^{2 g+2}, \\
C_{\mathrm{II}} & :=\left(c_{1} \cdot c_{2} \cdots c_{2 g}\right)^{4 g+2}, \quad \text { namely } C_{\mathrm{II}}=J^{2}, \\
P & :=d \cdot{ }_{W}\left(c_{g+1} \cdots c_{3} \cdot c_{2}\right) \cdots{ }_{W}\left(c_{2 g} \cdots c_{g+2} \cdot c_{g+1}\right) \\
& \quad\left(c_{g+1} \cdots c_{3} \cdot c_{2}\right) \cdots\left(c_{2 g} \cdots c_{g+2} \cdot c_{g+1}\right), \quad(g \text { is even })
\end{aligned}
$$

where $W:=\left(c_{1} \cdot c_{2} \cdots c_{g}\right)^{-(g+1)}$. When $g$ is even, the words $Q$ and $R$ are defined by

$$
\begin{aligned}
Q & :=\left(c_{1} \cdot c_{2} \cdots c_{2 g+1}\right)^{g+1} \cdot d \cdot W_{1}\left(c_{g+1}\right) \cdot W_{2}\left(c_{g+2}\right) \cdots W_{g+1}\left(c_{2 g+1}\right), \\
R & :=d \cdot{ }_{W_{1}}\left(c_{g+1}\right) \cdot{ }_{W_{2}}\left(c_{g+2}\right) \cdots W_{g+1}\left(c_{2 g+1}\right) \cdot\left(c_{2 g+1} \cdots c_{2} \cdot c_{1}\right)^{g+1},
\end{aligned}
$$


where $W_{i}:=\left(c_{i+g-1} \cdots c_{i+1} c_{i}\right)^{-1}$ for each $i \in\{1,2, \ldots, g+1\}$. When $g$ is odd, the words $Q$ and $R$ are defined by

$$
\begin{array}{r}
Q:=\left(c_{1} \cdot c_{2} \cdots c_{2 g+1}\right)^{g+2} \cdot d \cdot\left(c_{g-1} \cdot c_{2} \cdot c_{1}\right)^{2} \cdot W_{1}\left(c_{g}\right) \cdot W_{2}\left(c_{g+1}\right) \cdots W_{g+2}\left(c_{2 g+1}\right), \\
R:=c_{1} \cdot c_{2} \cdots c_{2 g+1} \cdot d \cdot\left(c_{g-1} \cdots c_{2} \cdot c_{1}\right)^{2} \cdot W_{1}\left(c_{g}\right) \cdot W_{2}\left(c_{g+1}\right) \cdots W_{g+2}\left(c_{2 g+1}\right) \\
\cdot\left(c_{2 g+1} \cdots c_{2} \cdot c_{1}\right)^{g+1},
\end{array}
$$

where $W_{i}:=c_{i}^{-1} c_{i+1}^{-1} \cdots c_{i+g-2}^{-1}$ for each $i \in\{1,2, \ldots, g+2\}$.

Endo [2008] proved that the words $I, J, C_{\mathrm{I}}, C_{\mathrm{II}}, P, Q$ and $R$, as products of positive Dehn twists, satisfy the following positive relations:

$$
\begin{aligned}
I^{2}=1, & C_{\mathrm{I}}=1, \quad C_{\mathrm{II}}=1, \\
P^{2}=1, & Q=1, \quad P I=1, \quad P J=1, \quad R I=1 \quad(g \text { is even }) \\
Q & =1, \quad R=1 \quad(g \text { is odd }) .
\end{aligned}
$$

From these positive relations but for $R I=1$, we can obtain nonminimal hyperelliptic Lefschetz fibrations admitting sections of square (-1); see [Endo 2008]. Of course, the three relations $I^{2}=1, C_{\mathrm{I}}=1$ and $C_{\mathrm{II}}=1$ are the same as the positive relations $W_{1}, W_{2}, W_{3}$.

\section{The geography of symplectic 4-manifolds}

Smooth closed 4-manifolds can be endowed with different structures as complex structures, almost-complex structures and symplectic structures.

A symplectic structure on a smooth 4-manifold $M$ is a closed 2-form $\omega$ that is nondegenerate as a bilinear form on each tangent space $T_{x} M$. A symplectic 4-manifold is a smooth 4-manifold $M$ together with a symplectic structure $\omega$.

An almost-complex structure on $M$ is a bundle endomorphism $J: T M \rightarrow T M$ of the tangent bundle $T M$ with $J^{2}=-i d_{T M}$. Since $(T M, J)$ is regarded as a $\mathbb{C}^{2}$-bundle over $M$, we can define Chern classes

$$
c_{1}(M, J):=c_{1}(T M, J), \quad c_{2}(M, J):=c_{2}(T M, J) .
$$

Furthermore, it is well-known that any symplectic 4-manifold $(M, \omega)$ admits an $\omega$-compatible almost-complex structure, which is an almost-complex structure $J$ such that $g(u, v):=\omega(u, J v)(u, v \in T M)$ is a Riemannian metric.

A smooth map $\varphi: \Sigma \rightarrow M$ from a possibly disconnected compact Riemann surface $(\Sigma, j)$ to $(M, J)$ is said to be $J$-holomorphic if the differential $d \varphi$ satisfies

$$
d \varphi \circ j=J \circ d \varphi .
$$

We call the image $\varphi(\Sigma)$ a J-holomorphic curve or a pseudoholomorphic curve with respect to $J$. If $C$ is a pseudoholomorphic curve with respect to an $\omega$-compatible 
almost complex structure, then $C$ is also $\omega$-symplectic. A pseudoholomorphic curve on a symplectic 4-manifold is one of the most important tools in modern symplectic 4-dimensional topology and has a lot in common with holomorphic curves. For example, two distinct pseudoholomorphic curves intersect discretely and positively. Hence, the algebraic intersection number between two distinct pseudoholomorphic curves stands for the geometric intersection number.

Many typical examples of simply connected 4-manifolds are given by complex surfaces. Since they are simply connected, they are also Kähler, that is, symplectic 4-manifolds admitting symplectic structures whose compatible almost-complex structures are integrable. The geography of simply connected minimal complex surfaces, that is, the range of Chern pairs $\left(c_{1}^{2}, c_{2}\right)$ of such complex surfaces, is as in Figure 1 . The boundary of the range is given by the Noether line $\left(5 c_{1}^{2}=c_{2}-36\right)$ and the Bogomolov-Miyaoka-Yau line $\left(c_{1}^{2}=3 c_{2}\right)$.

Symplectic 4-manifolds have a lot in common with complex surfaces: pseudoholomorphic curves play a role as holomorphic curves on complex surfaces. Donaldson's theorem (Theorem 2-2) gives us a symplectic version of the ample divisor. By Taubes' theorem [1995; 1996] on the Gromov-Witten invariants $\mathrm{Gr}_{T}$ and the canonical classes, we can regard a pseudoholomorphic representative of the canonical class $K_{M}$ of a symplectic 4-manifold $(M, \omega)$ as a symplectic version of the canonical divisor. Thus, one would like to achieve a similar classification as in complex surfaces for symplectic 4-manifolds. We consider the geography problem for symplectic 4-manifolds: Which pairs $(m, n)$ of integers are realized as the Chern pairs $\left(c_{1}^{2}, c_{2}\right)$ of a symplectic 4 -manifold?

We review Chern classes of symplectic 4-manifolds. We notice the following fundamental relations between $c_{1}^{2}$ and $c_{2}$ first:

$$
\begin{aligned}
& c_{1} \equiv w_{2}(\bmod 2), \\
& c_{1}^{2}=3 \sigma+2 e \quad(\text { Hirzebruch's signature theorem }), \\
& c_{2}=e .
\end{aligned}
$$

Thus, the pair $\left(c_{1}^{2}, c_{2}\right)$ is determined uniquely by the pair $(\sigma, e)$. Conversely, the pair $(\sigma, e)$ is determined uniquely by the pair $\left(c_{1}^{2}, c_{2}\right)$. Furthermore, since $c_{1}$ is characteristic, we have that $c_{1}^{2} \equiv w_{2}(\bmod 8)$. Hence, the Noether formula holds also for symplectic 4-manifolds:

$$
c_{1}^{2}+c_{2} \equiv 0(\bmod 12) .
$$

By use of a pseudoholomorphic representative of the canonical class $K_{M}$, one can prove part (1) of the following theorem:

Theorem 4-1 [Taubes 1996; Liu 1996]. (1) If $M$ is a minimal symplectic 4manifold with $b_{2}^{+}(M)>1$, then $c_{1}^{2}(M) \geq 0$. 
(2) Let $M$ be a minimal symplectic 4-manifold with $b_{2}^{+}(M)=1$. If $c_{1}^{2}(M)<0$, then $M$ must be an irrational ruled surface.

It follows from an easy calculation in (co)homology that, if $M$ is a symplectic 4-manifold with $b_{1}(M) \leq 1$, then $c_{1}^{2}(M) \leq 5 c_{2}(M)$. As for nonminimal symplectic 4-manifolds, we have

$$
c_{1}^{2}\left(M \# \overline{\mathbb{C P ^ { 2 }}}\right)=c_{1}^{2}(M)-1 \quad \text { and } \quad c_{2}\left(M \# \overline{\mathbb{C P ^ { 2 }}}\right)=c_{2}(M)+1 .
$$

Namely, the blow-up translates lattice points on the $\left(c_{2}, c_{1}^{2}\right)$-plane along the vector $(1,-1)$. Thus, the problem of the maximum for the slope $c_{1}^{2} / c_{2}$ comes to our mind. The author has no answer for this problem. However, it is expected that, if $M$ is a symplectic 4-manifold which is not an irrational ruled surface, then $c_{1}^{2}(M) \leq 3 c_{2}(M)$. See [Stipsicz 2000].

Since surfaces of general type are Kähler, it follows from the geography of complex surfaces that, for most of lattice points $(m, n)$ with $\frac{1}{5}(n-36) \leq m \leq 3 n$ and $m \geq 0$, there are symplectic 4-manifolds with $\left(c_{1}^{2}, c_{2}\right)=(m, n)$. On the other hand, Gompf [1995], Stipsicz [1996], and Fintushel and Stern [1998] showed that the existential range of $\left(c_{1}^{2}, c_{2}\right)$ of symplectic 4-manifolds is larger than that of complex surfaces.

As for the geography of Lefschetz fibrations, we can regard the geography of 4-manifolds admitting Lefschetz fibrations as one of symplectic 4-manifolds by the works of Donaldson and Gompf. Furthermore, we can also consider the geography of fibration structures of Lefschetz fibrations as follows:

Let $f: X \rightarrow S^{2}$ be a genus- $g$ Lefschetz fibration with $n_{0}$ irreducible singular fibers and $s_{h}$ reducible singular fibers of type $I I_{h}(1 \leq h \leq[g / 2])$. We denote the number of singular fibers of $f$ by $\mu(f):=n_{0}+\sum_{h=1}^{[g / 2]} s_{h}$. Then, we have the following:

$$
c_{1}^{2}(X)=3 \sigma(X)+2 e(X), \quad c_{2}(X)=e(X)=4-4 g+\mu(f) .
$$

Hence, by Theorem 2-5, we can calculate the Chern pairs $\left(c_{1}^{2}(X), c_{2}(X)\right)$ from the $([g / 2]+1)$-tuple $\left(n_{0}, s_{1}, \ldots, s_{[g / 2]}\right)$ of the numbers of singular fibers for a hyperelliptic genus- $g$ Lefschetz fibration $f: X \rightarrow S^{2}$. Thus, we regard the geography problem of (possibly nonhyperelliptic) genus- $g$ Lefschetz fibrations as characterizing the $([g / 2]+1)$-tuple $\left(n_{0}, s_{1}, \ldots, s_{[g / 2]}\right)$ of the numbers of singular fibers.

We now recall some facts about the number of singular fibers. The following inequalities hold for the number of irreducible singular fibers and the number of reducible singular fibers:

Theorem 4-2 [Stipsicz 1999]. Let $f: X \rightarrow S^{2}$ be a nontrivial genus-g Lefschetz fibration with $n_{0}$ irreducible singular fibers and $s_{h}$ reducible singular fibers of type $I_{h}(1 \leq h \leq[g / 2])$. 
(1) $5 n_{0} \geq \sum_{h=1}^{[g / 2]} s_{h}$.

(2) $n_{0}>0$, that is, there is no Lefschetz fibration with only reducible singular fibers.

Let $N(g)$ denote the minimal number of singular fibers in genus- $g$ Lefschetz fibrations over $S^{2}$, namely,

$$
N(g):=\min \left\{\mu(f) \mid f: X \rightarrow S^{2} \text { is a genus- } g \text { Lefschetz fibration }\right\} .
$$

Theorem 4-3 [Korkmaz and Ozbagci 2001; Stipsicz 1999]. We have estimates on $N(g)$ as follows:

(1) $N(2)=7$, or 8 .

(2) $N(g) \geq \frac{1}{5}(4 g+2)$.

Proposition 4-4 [Sato 2010b]. Let $f: X \rightarrow S^{2}$ be a genus-2 Lefschetz fibration with $\mu(f)$ singular fibers.

(1) If $\mu(f)=7$, then $X$ is diffeomorphic to $S^{2} \times T^{2} \# 3 \overline{\mathbb{C P}^{2}}$.

(2) If $\mu(f)=8$, then $X$ is diffeomorphic to $S^{2} \times T^{2} \# 4 \overline{\mathbb{C P}^{2}}$.

By considering the abelianization of the global monodromy of a Lefschetz fibration, we can obtain the congruence on the number of singular fibers. The following proposition is proved by noting that the abelianization $H_{1}\left(\Gamma_{2} ; \mathbb{Z}\right)$ of $\Gamma_{2}$ is isomorphic to the cyclic group of order 10 .

Proposition 4-5 [Persson 1992]. Suppose that a genus-2 Lefschetz fibration over $S^{2}$ has $n_{0}$ irreducible singular fibers and $s$ reducible singular fibers. Then, we have

$$
n_{0}+2 s \equiv 0(\bmod 10)
$$

If $g \geq 3$, then $H_{1}\left(\Gamma_{g} ; \mathbb{Z}\right)=0$, and so we can get no information on the number of singular fibers. However, if we consider the hyperelliptic case, then we can get information for hyperelliptic Lefschetz fibrations. Since the abelianization $H_{1}\left(\Gamma_{g}^{\text {hyp }} ; \mathbb{Z}\right)$ of the hyperelliptic mapping class group $\Gamma_{g}^{\text {hyp }}$ is isomorphic to $\mathbb{Z} / 2(2 g+1)$ if $g$ is even and $\mathbb{Z} / 4(2 g+1)$ if $g$ is odd, we obtain the congruence on the number of singular fibers of a hyperelliptic fibration.

Proposition 4-6 [Endo 2000]. Suppose that $f: X \rightarrow S^{2}$ is a genus- $g$ hyperelliptic Lefschetz fibration with $n_{0}$ irreducible singular fibers and $s_{h}$ singular fibers of type $I_{h}(h=1,2, \ldots,[g / 2])$. Then, we have

$$
n_{0}+4 \sum_{h=1}^{[g / 2]} h(2 h+1) s_{h} \equiv 0 \quad\left(\bmod \left\{\begin{array}{lc}
2(2 g+1) & \text { (if } g \text { is even }) \\
4(2 g+1) & \text { (if } g \text { is odd })
\end{array}\right\}\right) .
$$


Remark 4-7. By taking the fiber sums, we can construct genus- $g$ Lefschetz fibrations with arbitrarily large numbers of singular fibers. For example, for the genus- $g$ hyperelliptic Lefschetz fibration $f: \mathbb{C P}^{2} \#(4 g+5) \overline{\mathbb{C P}} \bar{C}^{2} \rightarrow \mathbb{C P}$ corresponding to the positive relation

$$
W_{1}:\left(c_{1} \cdot c_{2} \cdots c_{2 g} \cdot c_{2 g+1} \cdot c_{2 g+1} \cdot c_{2 g} \cdots c_{2} \cdot c_{1}\right)^{2}=1
$$

we consider the fiber sum $\#_{m F} f$ of $m$ copies of $f$. Then, the total space of $\#_{m F} f$ is minimal and $\#_{m F} f$ has $4(2 g+1) m$ irreducible singular fibers. Hence, the set of all $\left(n_{0}, s_{1}, \ldots, s_{[g / 2]}\right)$ of the numbers of singular fibers of genus- $g$ Lefschetz fibrations over $S^{2}$ is not bounded.

We shall consider the geography problem of Lefschetz fibrations in Section 6.

\section{The canonical classes of nonminimal Lefschetz fibrations over $S^{2}$}

2-spheres of square -1 in Lefschetzfibrations. Now we begin with two important theorems on smoothly embedded spheres in a symplectic 4-manifold with selfintersection number -1 .

Theorem 5-1 ((-1)-curve theorem, [Li and Liu 1995; Taubes 1996]). Let $(M, \omega)$ be a closed symplectic 4-manifold. Suppose that $M$ is neither the blow-up of a rational surface nor the blow-up of a ruled surface. Then, any smoothly embedded sphere of square -1 is $\mathbb{Z}$-homologous to a pseudoholomorphic rational curve of square -1 after the appropriate choice of an orientation of the sphere.

Taubes showed this theorem for $b_{2}^{+}(M)>1$, and Li and Liu showed this theorem for $b_{2}^{+}(M)=1$.

Theorem 5-2 [Ohta and Ono 2005]. Let $(M, \omega)$ be a closed symplectic 4-manifold and $F$ an irreducible pseudoholomorphic curve in $M$ with respect to an $\omega$-compatible almost-complex structure $J_{0}$. Suppose that the genus of $F$ is positive. Then, there exists an almost-complex structure $J$, which is arbitrarily close to $J_{0}$, such that $F$ and any symplectic sphere of square -1 are represented by J-holomorphic curves simultaneously.

Next we consider spheres of square -1 in Lefschetz fibrations. Let $f: X \rightarrow S^{2}$ be a nonminimal genus- $g$ Lefschetz fibration. Namely, we let $X$ admit smoothly embedded spheres of square -1 . Since we suppose that $g \geq 2, X$ has a symplectic structure $\omega$ with an $\omega$-compatible almost complex structure $J$ for which the fibers are pseudoholomorphic (Theorem 2-3). From now on, we assume that a Lefschetz fibration $f: X \rightarrow S^{2}$ is not minimal and $X$ admits such structures $\omega$ and $J$.

Let $E \in H^{2}(X ; \mathbb{Z})$ be the Poincaré dual of the homology class that is represented by a smoothly embedded sphere of square -1 in $X$. By changing the orientation of this sphere if necessary, we may assume that $E \cdot[\omega]>0$, because we have 
$E \cdot[\omega] \neq 0$ by the $(-1)$-curve theorem and the fact that $\omega_{\mid \Sigma}$ on a closed symplectic submanifold $\Sigma$ is a volume form of $\Sigma$. We denote by $\mathscr{E}_{X}$ the set of all the Poincaré duals of the homology classes $E$ that can be represented by smoothly embedded spheres of square -1 and satisfy $E \cdot[\omega]>0$. Moreover, let $F$ denote the Poincaré dual of the homology class represented by a generic fiber. Then, we have the following theorem:

Theorem 5-3 [Sato 2008]. Suppose that $X$ is neither the blow-up of a rational surface nor the blow-up of a ruled surface. We set $\mathscr{E}_{X}=\left\{E_{1}, E_{2}, \ldots, E_{m}\right\}$. Then:

(1) $E_{i} \cdot F \geq 1$ for any $E_{i} \in \mathscr{E}_{X}$,

(2) $m \leq\left(\sum_{i=1}^{m} E_{i}\right) \cdot F \leq 2 g-2$.

Remark 5-4. Suppose that $X$ is neither the blow-up of a rational surface nor the blow-up of a ruled surface. Then, by the (-1)-curve theorem, $E \in \mathscr{E}_{X}$ can be represented by an $\omega$-symplectic sphere of square -1 . Hence, it follows from Theorem 5-2 and the positivity of intersections of pseudoholomorphic curves that, if $E \in \mathscr{E}_{X}$ satisfies $E \cdot F=1$, then $E$ is represented by a (-1)-section of $f$.

Thus, by Theorem 5-3, we can classify $\mathscr{E}_{X}$ into several types. For example, $\mathscr{E}_{X}$ in the cases of $g=2$ and $g=3$ are classified as follows:

The case $g=2$ : We consider a genus-2 Lefschetz fibration $f: X \rightarrow S^{2}$ with spheres of square -1 . If $X$ is neither rational nor ruled, then Theorem 5-3 states that $\mathscr{E}_{X}$ is one of the following three:

Type $(1,1): \mathscr{E}_{X}=\left\{E_{1}, E_{2}\right\}, E_{1} \cdot F=E_{2} \cdot F=1$.

Type (1): $\mathscr{E}_{X}=\{E\}, E \cdot F=1$.

Type (2): $\mathscr{E}_{X}=\{E\}, E \cdot F=2$.

In the first and the second cases, spheres representing $\mathscr{E}_{X}$ are $(-1)$-sections of $f: X \rightarrow S^{2}$. Note that $E_{1} \cdot E_{2}=0$ for $E_{1}$ and $E_{2}$ in Type $(1,1)$, which follows from the proof of Corollary 3 in [Li 1999].

The case $g=3$ : We consider a genus-3 Lefschetz fibration $f: X \rightarrow S^{2}$ with spheres of square -1 . If $X$ is neither rational nor ruled, Theorem 5-3 states that the set $\mathscr{E}_{X}$ of spheres of square -1 is one of the following 11 types:

Type $(1,1,1,1): \mathscr{E}_{X}=\left\{E_{1}, E_{2}, E_{3}, E_{4}\right\}, E_{1} \cdot F=E_{2} \cdot F=E_{3} \cdot F=E_{4} \cdot F=1$.

Type $(1,1,2): \mathscr{\mathscr { E }}_{X}=\left\{E_{1}, E_{2}, E\right\}, E_{1} \cdot F=E_{2} \cdot F=1, E \cdot F=2$.

Type $(1,3): \mathscr{E}_{X}=\left\{E_{1}, E\right\}, E_{1} \cdot F=1, E \cdot F=3$.

Type $(2,2): \mathscr{E}_{X}=\left\{E_{1}, E_{2}\right\}, E_{1} \cdot F=E_{2} \cdot F=2$.

Type (4): $\mathscr{E}_{X}=\{E\}, E \cdot F=4$.

Type $(1,1,1): \mathscr{E}_{X}=\left\{E_{1}, E_{2}, E_{3}\right\}, E_{1} \cdot F=E_{2} \cdot F=E_{3} \cdot F=1$. 
Type $(1,2): \mathscr{E}_{X}=\left\{E_{1}, E\right\}, E_{1} \cdot F=1, E \cdot F=2$.

Type (3): $\mathscr{E}_{X}=\{E\}, E \cdot F=3$.

Type $(1,1): \mathscr{E}_{X}=\left\{E_{1}, E_{2}\right\}, E_{1} \cdot F=E_{2} \cdot F=1$.

Type (2): $\mathscr{\mathscr { E }}_{X}=\{E\}, E \cdot F=2$.

Type (1): $\mathscr{E}_{X}=\{E\}, E \cdot F=1$.

Furthermore, if we set $\sum \mathscr{E}_{X}:=\sum_{i=1}^{m} E_{i}$ for $\mathscr{E}_{X}=\left\{E_{1}, E_{2}, \ldots, E_{m}\right\}$, then types of $\mathscr{E}_{X}$ are shared as follows:

$$
\begin{aligned}
& \left(\sum \mathscr{E}_{X}\right) \cdot F=4: \text { Type }(1,1,1,1), \text { Type }(1,1,2), \text { Type }(1,3), \text { Type }(2,2), \\
& \left(\sum \mathscr{E}_{X}\right) \cdot F=3: \text { Type }(1,1,1), \text { Type }(1,2), \text { Type }(3) \\
& \left(\sum \mathscr{E}_{X}\right) \cdot F=2: \text { Type }(1,1), \text { Type }(2) \\
& \left(\sum \mathscr{E}_{X}\right) \cdot F=1: \text { Type }(1)
\end{aligned}
$$

In general, if the set $\mathscr{E}_{X}=\left\{E_{1}, E_{2}, \ldots, E_{m}\right\}$ for a nonminimal genus- $g$ Lefschetz fibration $f: X \rightarrow S^{2}$ satisfies the conditions

$$
E_{i} \cdot F=j_{i}, \quad j_{1} \leq j_{2} \leq \cdots \leq j_{m}
$$

then $\mathscr{E}_{X}$ is said to be of Type $\left(j_{1}, j_{2}, \ldots, j_{m}\right)$.

Now we can state the main theorem.

Theorem 5-5. Let $f: X \rightarrow S^{2}$ be a nonminimal genus-g Lefschetz fibration. Let $K_{X}$ be the canonical class of $(X, \omega)$. Suppose that $X$ is neither the blow-up of a rational surface nor the blow-up of a ruled surface. Then, the canonical class $K_{X}$ can be determined according to the types of $\mathscr{E}_{X}$ as follows:

[1] If $g=2$, we have:

(1) If $\mathscr{E}_{X}$ is of Type $(1,1)$, then $K_{X}=E_{1}+E_{2}$, where $\mathscr{E}_{X}=\left\{E_{1}, E_{2}\right\}$.

(2) If $\mathscr{E}_{X}$ is of Type (2), then $K_{X}=E$, where $\mathscr{E}_{X}=\{E\}$.

(3) If $\mathscr{E}_{X}$ is of Type (1), then $K_{X}=2 E+R$ or $K_{X}=2 E+F$. Here, $\mathscr{E}_{X}=\{E\}$ and $R$ is a genus-1 irreducible component of a reducible singular fiber such that $E \cdot R=1$. Moreover, in the case of $K_{X}=2 E+R$, the fibration $f$ has only one reducible singular fiber. In the case of $K_{X}=2 E+F$, the fibration $f$ has no reducible singular fiber.

[2] If $g \geq 3$, we have:

(1) If $\left(\sum \mathscr{E}_{X}\right) \cdot F=2 g-2$, then $K_{X}=\sum \mathscr{E}_{X}$. 
(2) If $\left(\sum \mathscr{E}_{X}\right) \cdot F=2 g-3$, we have

$$
K_{X}=2 E_{1}+\sum_{\substack{E \in_{\mathscr{E}} \\ E \neq E_{1}}} E+R .
$$

Here, $E_{1}$ is a (-1)-section of $f$ and $R$ is a genus-1 irreducible component of a reducible singular fiber such that $E_{1} \cdot R=1$ and $E \cdot R=0$ for any $E \in \mathscr{E}_{X}\left(E \neq E_{1}\right)$.

Proof. We can find out the proof in the case of $g=2$ in the proof of Theorem 5-1 of [Sato 2008]. We suppose that the fiber genus of $f$ is greater than two.

Equip $X$ with an almost complex structure $J$ such that fibers of $f$ are $J$ holomorphic curves. Let $\mathscr{E}_{X}=\left\{E_{1}, E_{2}, \ldots, E_{m}\right\}$ be the set of all cohomology classes represented by spheres of square -1 . Set $A=K_{X}-\sum \mathscr{E}_{X}=K_{X}-\sum_{i=1}^{m} E_{i}$. By the adjunction formula, we have $K_{X} \cdot F=2 g-2, K_{X} \cdot E_{i}=-1$ for any $i$ and so $A \cdot E_{i}=K_{X} \cdot E_{i}-E_{i}^{2}=0$ for any $i$. Furthermore, we have

$$
\begin{gathered}
A^{2}=A \cdot\left(K_{X}-\sum \mathscr{E}_{X}\right)=A \cdot K_{X}-\sum_{i=1}^{m} A \cdot E_{i}=A \cdot K_{X}, \\
A \cdot F=K_{X} \cdot F-\left(\sum \mathscr{E}_{X}\right) \cdot F=(2 g-2)-\left(\sum \mathscr{E}_{X}\right) \cdot F .
\end{gathered}
$$

Hence, if $\left(\sum \mathscr{E}_{X}\right) \cdot F$ is $2 g-2$ or $2 g-3$, then

$$
A \cdot F= \begin{cases}0 & \text { if }\left(\sum \mathscr{E}_{X}\right) \cdot F=2 g-2, \\ 1 & \text { if }\left(\sum \mathscr{E}_{X}\right) \cdot F=2 g-3 .\end{cases}
$$

Since each class $E_{i}$ of $\mathscr{E}_{X}$ is represented by a pseudoholomorphic curve and is a basic class of the Gromov-Taubes invariant $\mathrm{Gr}_{T}$ [Taubes 1995; 1996], it follows from the duality formula of the Gromov-Taubes invariant that $A$ is also a basic class, that is, $\operatorname{Gr}_{T}(A) \neq 0$. Hence, the class $A$ has a $J$-holomorphic representative $\mathscr{C}=\left\{\left(C_{j}, m_{j}\right)\right\}_{1 \leq j \leq n}$ such that each $C_{j}$ is a $J$-holomorphic curve and each $m_{j}$ $(\geq 1)$ is the multiplicity of $C_{j}$. The components $C_{j}$ of $\mathscr{C}$ are not always nonsingular.

(1) The case of $\left(\sum \mathscr{E}_{X}\right) \cdot F=2 g-2$ : The cohomology class $A$ is represented by $\mathscr{C}=\left\{\left(C_{j}, m_{j}\right)\right\}_{1 \leq j \leq n}$ and we have $A=\sum_{j=1}^{n} m_{j}\left[C_{j}\right]$. Since $A \cdot F=0$, we have $\sum_{j=1}^{n} m_{j}\left[C_{j}\right] \cdot F=0$. Noting that pseudoholomorphic curves have locally positive intersections, this implies that each component $C_{j}$ of $\mathscr{C}$ is contained in a fiber. Hence, we have $\left[C_{j}\right]^{2}=0$ or -1 . If $C_{j}$ is a generic fiber or an irreducible singular fiber, then $\left[C_{j}\right]^{2}=0$. If $C_{j}$ is a component of a reducible singular fiber, then $\left[C_{j}\right]^{2}=-1$. However, since $A \cdot E_{i}=0$ and $E_{i} \cdot F \neq 0$ for any $i$, each component $C_{j}$ is neither a generic fiber nor an irreducible singular fiber. Furthermore, since $f$ is relatively minimal, every fiber contains no sphere-component. Therefore, $C_{j}$ is a component of a reducible singular fiber with genus $\left(C_{j}\right) \geq 1$ and $\left[C_{j}\right]^{2}=-1$. If distinct components $C_{j}$ and $C_{k}$ intersected, they would be components of a 
reducible fiber and one of $C_{j}$ and $C_{k}$ would meet a section $E_{i}$. However, since $A \cdot E_{i}=0$, any component $C_{j}$ does not meet other components $C_{k}$.

Now we arrange the indices of components of $\mathscr{C}$. Let $C_{j, k}$ be a component of a reducible singular fiber such that the genus of $C_{j, k}$ is $k$. Let $m_{j, k}$ be the multiplicity of $C_{j, k}$. If $\mathscr{b}$ does not contain a component of genus $k$, then we set up a virtual component $C_{1, k}$ and $m_{1, k}=0$. Then, we have $A=\sum_{k=1}^{g-1} \sum_{j=1}^{n_{j}} m_{j, k}\left[C_{j, k}\right]$. Noting that $K_{X} \cdot\left[C_{j, k}\right]=2 k-1$ and $\left[C_{i, \ell}\right] \cdot\left[C_{j, k}\right]=0(k \neq \ell)$, we calculate $A^{2}$ and $A \cdot K_{X}$. We have

$$
\begin{aligned}
A^{2}= & \sum_{j=1}^{n_{1}} m_{j, 1}^{2}\left[C_{j, 1}\right]^{2}+\sum_{j=1}^{n_{2}} m_{j, 2}^{2}\left[C_{j, 2}\right]^{2}+\cdots+\sum_{j=1}^{n_{g-1}} m_{j, g-1}^{2}\left[C_{j, g-1}\right]^{2} \\
= & -\left(\sum_{j=1}^{n_{1}} m_{j, 1}^{2}+\sum_{j=1}^{n_{2}} m_{j, 2}^{2}+\cdots+\sum_{j=1}^{n_{g-1}} m_{j, g-1}^{2}\right), \\
A \cdot K_{X}= & \sum_{j=1}^{n_{1}} m_{j, 1}\left[C_{j, 1}\right] \cdot K_{X}+\sum_{j=1}^{n_{2}} m_{j, 2}\left[C_{j, 2}\right] \cdot K_{X}+\cdots \\
& +\sum_{j=1}^{n_{g-1}} m_{j, g-1}\left[C_{j, g-1}\right] \cdot K_{X} \\
= & \sum_{j=1}^{n_{1}} m_{j, 1}+3 \sum_{j=1}^{n_{g-1}} m_{j, 2}+\cdots+(2 g-3) \sum_{j=1}^{n_{g-1}} m_{j, g-1} .
\end{aligned}
$$

Hence, we have that $A^{2} \leq 0$ and $A \cdot K_{X} \geq 0$. Since $A^{2}=A \cdot K_{X}$, we have $A^{2}=A \cdot K_{X}=0$. Therefore, we have $m_{j, k}=0$ for any $j, k$, in particular $A=0$. Hence, $K_{X}=\sum \mathscr{E}_{X}$.

(2) The case of $\left(\sum \mathscr{E}_{X}\right) \cdot F=2 g-3$ : Since $A \cdot F=1$, the pseudoholomorphic representative $\mathscr{C}$ of $A$ contains a section $S$ as a component of $\mathscr{C}$. Then, we can see that $S$ is smooth and the multiplicity of $S$ is one. Suppose that $S$ is singular and $x \in S$ is a singular point of $S$. The fiber $F_{0}=f^{-1}(f(x))$ intersects $S$ at the singular point $x$. This fact implies that $[S] \cdot\left[F_{0}\right] \geq 2$, because pseudoholomorphic curves have locally positive intersections. However, this contradicts the fact that $A \cdot\left[F_{0}\right]=A \cdot F=1$. Hence, $S$ is a smooth section. Moreover, since $A \cdot F=1$, the multiplicity of $S$ is one.

Let $\left\{C_{j} \mid j=1,2, \ldots, n\right\}$ be the set of all components of $\mathscr{C}$ except $S$. Then, we can see that each $C_{j}$ contains in a fiber of $f$. Since $A=[S]+\sum_{j=1}^{n} m_{j}\left[C_{j}\right]$ and $A \cdot F=1$, we have that $1=A \cdot F=[S] \cdot F+\sum_{j=1}^{n} m_{j}\left[C_{j}\right] \cdot F=1+\sum_{j=1}^{n} m_{j}\left[C_{j}\right] \cdot F$ and $\sum_{j=1}^{n} m_{j}\left[C_{j}\right] \cdot F=0$. Hence, we have $\left[C_{j}\right] \cdot F=0$ for any $j$, and so each component $C_{j}$ contains in a fiber. 
Now we divide components of $\mathscr{C}$ except $S$ into generic/irreducible fibers and components of reducible fibers. Furthermore, we divide components of reducible fibers in $\mathscr{C}$ according to genera. Namely, the class $A$ is represented by the pseudoholomorphic curve $\mathscr{C}=\left\{(S, 1),\left(F_{i}, k_{i}\right),\left(C_{j, \ell}, m_{j, \ell}\right)\right\}$, where each $F_{i}$ is a generic fiber or an irreducible singular fiber and each $C_{j, \ell}$ is a component of a reducible singular fiber such that the genus of $C_{j, \ell}$ is $\ell$. Of course, we have that $\left[C_{j, \ell}\right]^{2}=-1$, $\left[C_{i, k}\right] \cdot\left[C_{j, \ell}\right]=0((i, k) \neq(j, \ell)), F \cdot\left[C_{j, \ell}\right]=0$ and $\left[C_{j, \ell}\right] \cdot K_{X}=2 \ell-1$. Since an irreducible singular fiber is homologous to the generic fiber $F$, components of $\mathscr{b}$ which are generic fibers or irreducible singular fibers yield the homology class $m F$, and so the class $A$ is given by

$$
\begin{aligned}
A & =[S]+m F+\sum_{j=1}^{n_{1}} m_{j, 1}\left[C_{j, 1}\right]+\sum_{j=1}^{n_{2}} m_{j, 2}\left[C_{j, 2}\right]+\cdots+\sum_{j=1}^{n_{g-1}} m_{j, g-1}\left[C_{j, g-1}\right] \\
& =[S]+m F+\sum_{\ell=1}^{g-1} \sum_{j=1}^{n_{\ell}} m_{j, \ell}\left[C_{j, \ell}\right] .
\end{aligned}
$$

We compare $A^{2}$ with $A \cdot K_{X}$ in the same way as the case (1). We have

$$
\begin{aligned}
A^{2}= & {[S]^{2}+m^{2} F^{2}+\sum_{\ell=1}^{g-1} \sum_{j=1}^{n_{\ell}} m_{j, \ell}^{2}\left[C_{j, \ell}\right]^{2}+2 m[S] \cdot F } \\
& +2 \sum_{\ell=1}^{g-1} \sum_{j=1}^{n_{\ell}} m_{j, \ell}[S] \cdot\left[C_{j, \ell}\right]+2 \sum_{\ell=1}^{g-1} \sum_{j=1}^{n_{\ell}} m_{j, \ell} F \cdot\left[C_{j, \ell}\right] \\
= & {[S]^{2}-\sum_{\ell=1}^{g-1} \sum_{j=1}^{n_{\ell}} m_{j, \ell}^{2}+2 m+2 \sum_{\ell=1}^{g-1} \sum_{j=1}^{n_{\ell}} m_{j, \ell}[S] \cdot\left[C_{j, \ell}\right], } \\
A \cdot K_{X}= & {[S] \cdot K_{X}+m F \cdot K_{X}+\sum_{\ell=1}^{g-1} \sum_{j=1}^{n_{\ell}} m_{j, \ell}\left[C_{j, \ell}\right] \cdot K_{X} } \\
= & -2-[S]^{2}+2 m(g-1)+\sum_{\ell=1}^{g-1} \sum_{j=1}^{n_{\ell}}(2 \ell-1) m_{j, \ell} .
\end{aligned}
$$

Since $A^{2}-A \cdot K_{X}=0$, we have

$(5-6)-2\left(1+[S]^{2}\right)+2 m(g-1)+\sum_{\ell=1}^{g-1} \sum_{j=1}^{n_{\ell}} m_{j, \ell}\left(2 \ell-1+m_{j, \ell}-2[S] \cdot\left[C_{j, \ell}\right]\right)=0$.

By Lemma 2.1 of [Stipsicz 2001], the self-intersection number of $S$ is negative, and so we have $-2\left(1+[S]^{2}\right) \geq 0$. Furthermore, since $S$ is a section, the intersection 
number $[S] \cdot\left[C_{j, \ell]}\right.$ is 0 or 1 . Hence, we have that $m_{j, \ell}\left(2 \ell-1+m_{j, \ell}-2[S] \cdot\left[C_{j, \ell}\right]\right) \geq 0$. Hence, each term in the left side of (5-6) is nonnegative. Therefore, we obtain

$$
\left\{\begin{array}{l}
-2\left(1+[S]^{2}\right)=0, \\
2 m(g-1)=0, \\
m_{j, \ell}\left(2 \ell-1+m_{j, \ell}-2[S] \cdot\left[C_{j, \ell}\right]\right)=0 \text { for any } j, \ell .
\end{array}\right.
$$

If $\ell \geq 2$, then we have $2 \ell-1+m_{j, \ell}-2[S] \cdot\left[C_{j, \ell}\right] \geq 1$ since $0 \leq[S] \cdot\left[C_{j, \ell}\right] \leq 1$. Hence it follows from the third equation above that $m_{j, \ell}=0$ for $\ell \geq 2$. Thus the pseudoholomorphic representative $\mathscr{C}$ consists only of the section $S$ and torus components of reducible singular fibers. Noting that $[S] \cdot\left[C_{j, 1}\right]$ is 0 or 1 , it follows that if $m_{j, 1}\left(1+m_{j, 1}-2[S] \cdot\left[C_{j, 1}\right]\right)=0$ and $m_{j, 1} \neq 0$, then $m_{j, 1}=1$ and $[S] \cdot\left[C_{j, 1}\right]=1$. On the other hand, since $[S]^{2}=-1$, the smooth section $S$ is a sphere of square -1 and the class $[S]$ is a member of $\mathscr{E}_{X}$. Set $E_{1}=[S]$.

Now we consider the case where there is a torus component in $\mathscr{C}$. Then, such a torus component meets the section $S$ and its multiplicity is one. Thus, we can write $A$ as $A=E_{1}+\sum_{j=1}^{n_{1}}\left[C_{j, 1}\right]$. Suppose that $n_{1} \geq 2$. Since $A \cdot E_{1}=0$ and $[S] \cdot\left[C_{j, 1}\right]=1$, we have that $n_{1}-1=\sum_{j \neq 1}\left[C_{j, 1}\right] \cdot E_{1}=A \cdot E_{1}-\left(E_{1}^{2}+\left[C_{1,1}\right] \cdot E_{1}\right)=0$. This is a contradiction. Hence, the class $A$ is written as $A=E_{1}+\left[C_{1,1}\right]$. Therefore, we obtain

$$
K_{X}=2 E_{1}+\sum_{\substack{E \in \mathscr{C}_{X} \\ E \neq E_{1}}} E+R
$$

where $R=\left[C_{1,1}\right]$ is the class represented by a torus component of a reducible singular fiber and $E_{1} \cdot R=1$. Since $C_{1,1}$ is a torus, we have $K_{X} \cdot R=1$ and so $\sum_{E \in \mathscr{C}_{X}, E \neq E_{1}} E \cdot R=K_{X} \cdot R-\left(2 E_{1} \cdot R+R^{2}\right)=0$. Hence, it follows from the positiveness of local intersections of pseudoholomorphic curves that $E \cdot R=0$ for any $E \in \mathscr{E}_{X}$ except $E_{1}$.

Next we consider the case where there is no torus component in $\mathscr{b}$. Then, we can write $A$ as $A=E_{1}$. Hence, we obtain

$$
K_{X}=2 E_{1}+\sum_{\substack{E \in \mathscr{C}_{X} \\ E \neq E_{1}}} E
$$

Then, the minimal model $X_{\min }$ of $X$ must satisfy that $K_{\min }^{2}<0$. Here, $K_{\min }$ denotes the canonical class of $X_{\min }$. However, since $X$ is not the blow-up of a ruled surface, it follows from Theorem 4-1 that $K_{\min }^{2}$ must be nonnegative. This is a contradiction. Hence, the case of $K_{X}=2 E_{1}+\sum_{E \in \mathscr{E}_{X}}, E \neq E_{1} E$ cannot occur. This completes the proof.

Remark 5-7. (1) If a nonminimal genus- $g$ Lefschetz fibration $f: X \rightarrow S^{2}$ satisfies $g \geq 3$ and $\mathscr{E}_{X}$ is of class $\left(\sum \mathscr{E}_{X}\right) \cdot F=2 g-3$, then $f$ must have some reducible singular fibers. 
(2) $\mathscr{E}_{X}$ of class $\left(\sum \mathscr{E}_{X}\right) \cdot F=2 g-3$ must contain some sections of square -1 . For example, the set $\mathscr{E}_{X}$ of type (3) does not appear for nonminimal genus-3 Lefschetz fibrations.

(3) The square of the canonical class $K_{X}$ for $X$ in Theorem 5-5 is bounded. In fact, when $\left(\sum \mathscr{E}_{X}\right) \cdot F=2 g-2$, we have that $2-2 g \leq K_{X}^{2} \leq-1$. When $\left(\sum \mathscr{E}_{X}\right) \cdot F=$ $2 g-3$, we have that $3-2 g \leq K_{X}^{2} \leq-2$.

The symplectic Kodaira dimension of nonminimal Lefschetz fibrations. Given any smooth complex surface $X$, there are four possibilities of the behavior of the plurigenera $P_{n}(X)$. The Kodaira dimension $\kappa(X)$ of $X$ is defined according to four possibilities: $\kappa(X)=-\infty, 0,1$, or 2 . It is well-known that smooth compact complex surfaces are classified in terms of the Kodaira dimension.

The first notion of the symplectic version of the Kodaira dimension appeared in [McDuff and Salamon 1996] and the symplectic Kodaira dimension is defined and discussed in detail in [Li 2006].

Definition 5-8. Let $(M, \omega)$ be a minimal symplectic 4-manifold with symplectic canonical class $K_{\omega}$. Then, the symplectic Kodaira dimension $\kappa^{s}(M, \omega)$ is defined as follows:

$$
\kappa^{s}(M, \omega)= \begin{cases}-\infty & \text { if } K_{\omega} \cdot[\omega]<0 \text { or } K_{\omega}^{2}<0, \\ 0 & \text { if } K_{\omega} \cdot[\omega]=0 \text { and } K_{\omega}^{2}=0, \\ 1 & \text { if } K_{\omega} \cdot[\omega]>0 \text { and } K_{\omega}^{2}=0, \\ 2 & \text { if } K_{\omega} \cdot[\omega]>0 \text { and } K_{\omega}^{2}>0 .\end{cases}
$$

The symplectic Kodaira dimension of a nonminimal 4-manifold $(M, \omega)$ is defined to be that of any minimal model of $(M, \omega)$.

Theorem 5-9 ([Li 2006]; see also [Dorfmeister and Zhang 2009]). Let $(M, \omega)$ be a closed symplectic 4-manifold with symplectic canonical class $K_{\omega}$. If $(M, \omega)$ is minimal, then:

(1) With $M$ given the orientation compatible with $\omega$, the symplectic Kodaira dimension of $(M, \omega)$ only depends on the oriented diffeomorphism type of $M$, that is, if $\omega^{\prime}$ is another symplectic form on $M$ compatible with the orientation of $M$, then $\kappa^{s}(M, \omega)=\kappa^{s}\left(M, \omega^{\prime}\right)$.

$$
\kappa^{s}(M, \omega)= \begin{cases}-\infty & \text { if } M \text { is rational or ruled, } \\ 0 & \text { if } K_{\omega} \text { is torsion, } \\ 1 & \text { if } K_{\omega} \text { is nontorsion but } K_{\omega}^{2}=0, \\ 2 & \text { if } K_{\omega}^{2}>0 .\end{cases}
$$

Hence, by Theorem 5-9(2), we can calculate the symplectic Kodaira dimension of $(M, \omega)$ in terms of the canonical class $K_{\omega}$. 
Now, we have two kinds of Kodaira dimension. In the case where $(M, \omega)$ admits a complex structure $J$, the equivalence of these Kodaira dimensions $\kappa(M, J)$ and $\kappa^{s}(M, \omega)$ was proved by $\mathrm{Li}$ :

Theorem 5-10 (Li; see [Dorfmeister and Zhang 2009, Theorem 3.1]). Let $M$ be a smooth 4-manifold that admits a symplectic structure $\omega$ as well as a complex structure $J$. Then, we have $\kappa^{s}(M, \omega)=\kappa(M, J)$.

Remark 5-11. There are smooth 4-manifolds $M$ that admits a symplectic structure $\omega$ and a complex structure $J$ but whose $(M, \omega, J)$ is not Kähler. For example, Kodaira-Thurston manifolds are such manifolds. Theorem 5-10 states that the equivalence of Kodaira dimensions $\kappa(M, J)$ and $\kappa^{s}(M, \omega)$ holds for not only the Kähler case but also the non-Kähler case.

For a nonminimal genus- $g$ Lefschetz fibration $f: X \rightarrow S^{2}$ with

$$
\left(\sum \mathscr{E}_{X}\right) \cdot F=2 g-2 \text { or }\left(\sum \mathscr{E}_{X}\right) \cdot F=2 g-3,
$$

we calculate the square $K_{\min }^{2}$ of the canonical class $K_{\min }$ of a minimal model of $X$ by Theorem 5-5. Furthermore, we can calculate the symplectic Kodaira dimension from $K_{\min }^{2}$.

Theorem 5-12. Let $f: X \rightarrow S^{2}$ be a nonminimal genus- $g$ Lefschetz fibration. Equip $X$ with the natural symplectic structure $\omega$ given by Theorem 2-3. Let $K_{X}$ be the canonical class of $(X, \omega)$ and $K_{\min }$ the canonical class of a minimal model of $X$. Suppose that $X$ is neither the blow-up of a rational surface nor the blow-up of a ruled surface.

[1] If $g=2$, we have:

(1) If $\mathscr{E}_{X}$ is of Type $(1,1)$, then $K_{\min }^{2}=0$ and $\kappa^{s}(X, \omega)=0$.

(2) If $\mathscr{E}_{X}$ is of Type (2), then $K_{\min }^{2}=0$ and $\kappa^{s}(X, \omega)=0$.

(3) If $\mathscr{E}_{X}$ is of Type (1), then $K_{\min }^{2}=0$ and $\kappa^{s}(X, \omega)=1$ when $K_{X}=2 E+R$. We have $K_{\min }^{2}=1$ and $\kappa^{s}(X, \omega)=2$ when $K_{X}=2 E+F$.

[2] If $g \geq 3$, we have:

(1) If $\left(\sum \mathscr{E}_{X}\right) \cdot F=2 g-2$, then $K_{\min }^{2}=0$ and $\kappa^{s}(X, \omega)=0$.

(2) If $\left(\sum \mathscr{E}_{X}\right) \cdot F=2 g-3$, then $K_{\min }^{2}=0$ and $\kappa^{s}(X, \omega)=1$.

For example, Tables 1 and 2 summarize the canonical class $K_{X}$ and the symplectic Kodaira dimension $\kappa^{s}(X)$ for nonminimal Lefschetz fibrations of fiber genus 2 or 3 .

The author has also investigated the Iitaka $D$-dimension of the adjoint divisor $K_{X}+F$ of $F$ for holomorphic Lefschetz fibrations. See [Sato 2010a].

Now we can state about the relationship between the Kodaira dimension and the base loci of Lefschetz pencils. 


\begin{tabular}{|cccc|}
\hline$\left(\sum \mathscr{E}_{X}\right) \cdot F$ & $\mathscr{E}_{X}$ & $K_{X}$ & $\kappa^{s}$ \\
\hline$\left(\sum \mathscr{E}_{X}\right) \cdot F=2$ & Type $(1,1)$ & $K_{X}=E_{1}+E_{2}$ & \\
& Type (2) & $K_{X}=E$ & $\kappa^{s}=0$ \\
$\left(\sum \mathscr{E}_{X}\right) \cdot F=1$ & Type (1) & $K_{X}=2 E+R$ & $\kappa^{s}=1$ \\
& & $K_{X}=2 E+F$ & $\kappa^{s}=2$ \\
\hline
\end{tabular}

Table 1. The canonical class and the Kodaira dimension of nonminimal genus-2 Lefschetz fibrations.

\begin{tabular}{|cccc|}
\hline$\left(\sum \mathscr{E}_{X}\right) \cdot F$ & $\mathscr{E}_{X}$ & $K_{X}$ & $\kappa^{s}$ \\
\hline & Type $(1,1,1,1)$ & $K_{X}=E_{1}+E_{2}+E_{3}+E_{4}$ & $\kappa^{s}=0$ \\
& Type $(1,1,2)$ & $K_{X}=E_{1}+E_{2}+E$ & $\kappa^{s}=0$ \\
$\left(\sum \mathscr{E}_{X}\right) \cdot F=4$ & Type $(1,3)$ & $K_{X}=E_{1}+E$ & $\kappa^{s}=0$ \\
& Type $(2,2)$ & $K_{X}=E_{1}+E_{2}$ & $\kappa^{s}=0$ \\
& Type (4) & $K_{X}=E$ & $\kappa^{s}=0$ \\
\hline & Type $(1,1,1)$ & $K_{X}=2 E_{1}+E_{2}+E_{3}+R$ & $\kappa^{s}=1$ \\
$\left(\sum \mathscr{E}_{X}\right) \cdot F=3$ & Type (1,2) & $K_{X}=2 E_{1}+E+R$ & $\kappa^{s}=1$ \\
& Type (3) & no existence & no existence \\
\hline
\end{tabular}

Table 2. The canonical class and the Kodaira dimension of nonminimal genus-3 Lefschetz fibrations.

Corollary 5-13. Let $(X, \omega)$ be a minimal symplectic 4-manifold that is neither rational nor ruled. Suppose that $X$ admits a Lefschetz pencil whose fiber genus $g$ is more than 2.

(1) If the base locus consists of $2 g-2$ base points, then the symplectic Kodaira dimension of $X$ is 0 .

(2) If the base locus consists of $2 g-3$ base points, then the symplectic Kodaira dimension of $X$ is 1 .

(3) If $X$ is a minimal symplectic 4-manifold with $\kappa^{s}(X)=2$ (for example, a minimal complex surface of general type), then the base locus consists of at most $2 g-4$ base points.

Proof. Suppose that $X$ admits a Lefschetz pencil whose base locus consists of $k$ base points. By blowing up at these $k$ base points, we obtain a nonminimal genus- $g$ Lefschetz fibration $Y=X \# k \overline{\mathbb{C} P^{2}} \rightarrow S^{2}$ with $k$ sections of square -1 . The set $\mathscr{E}_{Y}$ is of Type $(1,1, \ldots, 1)$ with $\left(\sum \mathscr{E}_{Y}\right) \cdot F=k$. Therefore, the claim is proved by Theorem 5-12.

Remark 5-14. (1) For any $g(\geq 1)$, a $K 3$ surface admits a Lefschetz pencil whose 
fiber genus is $g$ and whose base locus consists of $2 g-2$ base points. See [Smith 2001b].

(2) Auroux [2006a] calculated the monodromies of the canonical Lefschetz pencils on a pair of homeomorphic Horikawa surfaces $X_{1}$ and $X_{2}$. The Horikawa surface $X_{1}$ is a double cover of $\mathbb{C} \mathrm{P}^{1} \times \mathbb{C P}^{1}$ branched along a smooth algebraic curve of bidegree $(6,12)$. The Horikawa surface $X_{2}$ is a double cover of the Hirzebruch surface $\mathbb{F}_{6}$ branched along $\triangle_{-6} \cup C$, where $\triangle_{-6}$ is the exceptional section of $\mathbb{F}_{6}$ and $C$ is a smooth algebraic curve in the linear system $\left|5 \triangle_{6}\right|$. By the construction of $X_{1}$ and $X_{2}$, these Horikawa surfaces admit genus-2 Lefschetz fibrations with 120 singular fibers; the corresponding positive relations are

$$
\left(c_{1} \cdot c_{2} \cdot c_{3} \cdot c_{4} \cdot c_{5}^{2} \cdot c_{4} \cdot c_{3} \cdot c_{2} \cdot c_{1}\right)^{12}=1 \quad \text { and } \quad\left(c_{1} \cdot c_{2} \cdot c_{3} \cdot c_{4}\right)^{30}=1,
$$

respectively. See also [Fuller 1998]. Since these have fiber-sum decompositions, $X_{1}$ and $X_{2}$ are minimal by Stipsicz's conjecture. Furthermore, the Kodaira dimension of $X_{1}$ and $X_{2}$ is 2. Auroux showed that $X_{1}$ and $X_{2}$ admit genus-17 Lefschetz pencils with 16 base points.

(3) A surface $S$ of general type embedded in a higher dimensional projective space $\mathbb{C P}^{N}$ has a Lefschetz pencil. The intersections of $S$ with hyperplane sections $\left\{H_{t} \mid\right.$ $\left.t \in \mathbb{C P}{ }^{1}\right\}$ containing a generic linear subspace $A$ of complex codimension 2 make the family of curves, which give $S$ a Lefschetz pencil. If $[S]=r\left[\mathbb{C P}^{2}\right] \in H_{4}\left(\mathbb{C P}{ }^{N} ; \mathbb{Z}\right)$, then the base locus $B=S \cap A$ of the pencil consists on $r$ points. By noting the Enriques-Kodaira classification of complex surfaces, the genus $g$ of the generic fiber must be at least 2 . On the other hand, we have that $g \geq(r+4) / 2$ by Corollary 5-13.

Endo's question. By generalizing Chakiris' way to construct holomorphic genus-2 Lefschetz fibrations, Endo [2008] gave examples of hyperelliptic Lefschetz fibrations of arbitrary genus. We can find many examples of nonminimal hyperelliptic Lefschetz fibrations [ibid.].

For a positive relator $W$, we denote the Lefschetz fibration associated to the positive relation $W=1$ by $M_{W} \rightarrow S^{2}$. If $g$ is even, then each of $M_{P^{2}}, M_{Q}$, $M_{P I}$ and $M_{P J}$ in Section 3 is nonminimal. In the case of $g=2$, the 4-manifolds $M_{P J}, M_{R I}$ and $K 3 \# \overline{\mathbb{C P}}$ are homeomorphic to $3 \mathbb{C P}^{2} \# 20 \overline{\mathbb{C P}^{2}}$ by Freedman's classification theorem. Furthermore, $M_{P J}$ for $g=2, K 3 \# \overline{\mathbb{C P}}$ and $3 \mathbb{C} P^{2} \# 20 \overline{\mathbb{C P}^{2}}$ are mutually nondiffeomorphic. On the other hand, the manifold $M_{R I}$ for $g=2$ is not diffeomorphic to $3 \mathbb{C} \mathrm{P}^{2} \# 20 \overline{\mathbb{C P}}$. Hence, the following questions are natural.

Question 5-15 [Endo 2008, Problem 4.13]. Let $P J$ and $R I$ be the positive relators introduced on page 204.

(1) Determine whether $M_{P J}$ and $M_{R I}$ are diffeomorphic or not when $g=2$.

(2) Is $M_{R I}$ diffeomorphic to $K 3 \# \overline{\mathbb{C} P^{2}}$ ? 
Remark 5-16. The Lefschetz fibration $M_{R I} \rightarrow S^{2}$ for $g=2$ is isomorphic to the genus-2 Lefschetz fibration constructed by Auroux [2003], whose set of spheres of square -1 is of Type (2). See also [Sato 2008]. Hence, $M_{R I} \rightarrow S^{2}$ for $g=2$ admits no section of square -1 . Since $M_{P J} \rightarrow S^{2}$ for $g=2$ admits a section of square -1 , two genus-2 Lefschetz fibrations $M_{P J} \rightarrow S^{2}$ and $M_{R I} \rightarrow S^{2}$ are not isomorphic. Question 5-15(1) is whether $M_{P J}$ and $M_{R I}$ are diffeomorphic as manifolds.

In order to answer this question, we note the following proposition:

Proposition 5-17. Let $(M, \omega)$ and $\left(M^{\prime}, \omega^{\prime}\right)$ be minimal symplectic 4-manifolds. Suppose that the Kodaira dimension $\kappa^{s}(M, \omega)$ of $(M, \omega)$ is equal to 0 . If

$$
\kappa^{s}\left(M^{\prime}, \omega^{\prime}\right) \neq 0
$$

then $M^{\prime}$ is not orientation-preserving diffeomorphic to $M$.

Proof. Suppose there exists an orientation-preserving diffeomorphism $f: M \rightarrow M^{\prime}$. Since the isomorphism $f^{*}: H^{2}\left(M^{\prime} ; \mathbb{Z}\right) \rightarrow H^{2}(M ; \mathbb{Z})$ gives a one-to-one correspondence

$$
\mathscr{B} a s\left(M^{\prime}\right) \rightarrow \mathscr{B a s}(M),
$$

if $\mathscr{B} a s\left(M^{\prime}\right)$ has a nontorsion class, then $\mathscr{B} a s(M)$ has also a nontorsion class. Here, $\mathscr{B} a s(M)$ and $\mathscr{B} a s\left(M^{\prime}\right)$ denote the set of Seiberg-Witten basic classes of $M$ and $M^{\prime}$, respectively. By Theorem 5-9, the canonical class $K_{\omega} \in \mathscr{B} a s(M)$ is a torsion class. Furthermore, it follows from a theorem of Taubes [1994] (Theorem 10.1.11 of [Gompf and Stipsicz 1999]) that

$$
|K \cdot[\omega]| \leq\left|K_{\omega} \cdot[\omega]\right|=0
$$

for any $K \in \mathscr{B a s}(M)$. Hence, $|K \cdot[\omega]|=\left|K_{\omega} \cdot[\omega]\right|=0$ and $\mathscr{B a s}(M)=\left\{ \pm K_{\omega}\right\}$. In particular, $\mathscr{B} a s(M)$ contains only torsion classes. However, since $\kappa^{s}\left(M^{\prime}, \omega^{\prime}\right) \neq 0$, the canonical class $K_{\omega^{\prime}}$ is nontorsion, and so the set $\mathscr{B} a s\left(M^{\prime}\right)$ contains a nontorsion class. This is a contradiction.

Answer to Question 5.1. (1) The manifold $M_{P J}$ is not orientation-preserving diffeomorphic to the manifold $M_{R I}$.

(2) The manifold $M_{R I}$ is diffeomorphic to $K 3 \# \overline{\mathbb{C P ^ { 2 }}}$.

Proof. (1) The genus-2 Lefschetz fibration $M_{P J} \rightarrow S^{2}$ is of Type (1) and the genus-2 Lefschetz fibration $M_{R I} \rightarrow S^{2}$ is of Type (2). Let $M_{P J \min }$ and $M_{R I \min }$ be minimal models of $M_{P J}$ and $M_{R I}$, respectively. By Theorem 5-12, we have that $\kappa^{s}\left(M_{P J \min }\right)=1$ and $\kappa^{s}\left(M_{R I \min }\right)=0$. Therefore, it follows from Proposition 5-17 that $M_{P J \min }$ and $M_{R I \min }$ are not mutually orientation-preserving diffeomorphic. Since $M_{P J}$ and $M_{R I}$ are not the blow-ups of a rational surface nor the blow-ups of a ruled surface, two manifolds $M_{P J}$ and $M_{R I}$ are not orientation-preserving diffeomorphic by the uniqueness of minimal models of symplectic 4-manifolds. 
(2) Since the Lefschetz fibration $M_{R I} \rightarrow S^{2}$ for $g=2$ is isomorphic to the genus2 Lefschetz fibration constructed by Auroux, $M_{R I}$ is a simply connected Kähler 4-manifold. Furthermore, we have that $\kappa\left(M_{R I \min }\right)=\kappa^{s}\left(M_{R I \min }\right)=0$. Hence, $M_{R I \min }$ is a $\mathrm{K} 3$ surface. Noting the uniqueness of minimal models of symplectic 4-manifolds, $M_{R I}$ is diffeomorphic to $K 3 \# \overline{\mathbb{C P}}$.

\section{The geography of nonminimal Lefschetz fibrations over $S^{2}$}

The geography of nonminimal hyperelliptic Lefschetz fibrations. In this section, we consider the geography problem of genus- $g$ Lefschetz fibrations as characterizing the $([g / 2]+1)$-tuple $\left(n_{0}, s_{1}, \ldots, s_{[g / 2]}\right)$ of the numbers of singular fibers.

Then, we have the finiteness theorem of the geography of nonminimal hyperelliptic Lefschetz fibrations:

Theorem 6-1. Suppose that $X$ is neither the blow-up of a rational surface nor the blow-up of a ruled surface. Then, only finitely many $\left(n_{0}, s_{1}, \ldots, s_{[g / 2]}\right)$ can be realized as the $([\mathrm{g} / 2]+1)$-tuple of the numbers of singular fibers for nonminimal hyperelliptic genus- $g(\geq 2)$ Lefschetz fibrations with $\left(\sum \mathscr{E}_{X}\right) \cdot F=2 g-2,2 g-3$. Proof. For a hyperelliptic genus- $g$ Lefschetz fibration $f: X \rightarrow S^{2}$ with the $([g / 2]+1)$-tuple $\left(n_{0}, s_{1}, \ldots, s_{[g / 2]}\right)$ of the numbers of singular fibers, we can calculate $K_{X}^{2}$ from the number of singular fibers as follows:

$$
\begin{aligned}
K_{X}^{2} & =3 \sigma(X)+2 e(X) \\
& =\frac{g-1}{2 g+1} n_{0}+\sum_{h=1}^{[g / 2]}\left(\frac{12 h(g-h)-(2 g+1)}{2 g+1}\right) s_{h}+8(1-g) .
\end{aligned}
$$

Since $12 h(g-h)-(2 g+1)=-12(h-g / 2)^{2}+(3 g+1)(g-1)>0$ for $1 \leq h \leq[g / 2]$, we have

$$
\frac{g-1}{2 g+1}>0 \text { and } \quad \frac{12 h(g-h)-(2 g+1)}{2 g+1}>0 .
$$

Hence, every coefficient of $K_{X}^{2}=K_{X}^{2}\left(n_{0}, s_{1}, \ldots, s_{[g / 2]}\right)$ is positive. Therefore, since $K_{X}^{2}$ is bounded by Remark $5-7(3)$, the number of the $([g / 2]+1)$-tuple $\left(n_{0}, s_{1}, \ldots, s_{[g / 2]}\right)$ satisfying $K_{K}^{2}$ and the estimation in Section 4 is finite. In fact, since $K_{X}^{2} \leq-1$, we have

$$
\frac{g-1}{2 g+1} n_{0}+\sum_{h=1}^{[g / 2]}\left(\frac{12 h(g-h)-(2 g+1)}{2 g+1}\right) s_{h}+8(1-g) \leq-1 .
$$

From the above inequality, we obtain

$$
n_{0} \leq \frac{(2 g+1)(8 g-9)}{g-1} .
$$


Moreover, noting that the number $n_{0}$ of irreducible singular fibers is positive [Stipsicz 1999], we have

$$
s_{h} \leq \frac{16 g^{2}-11 g-8}{12 h(g-h)-(2 g+1)}
$$

for any $h(1 \leq h \leq[g / 2])$.

From Theorem 6-4, we can obtain an estimate for $\mu(f)=n_{0}+\sum_{h=1}^{[g / 2]} s_{h}$ and a partial answer for Smith's question (Question 1-1).

Corollary 6-2. There is an upper bound on the number of singular fibers of nonminimal hyperelliptic genus- $g(\geq 3)$ Lefschetz fibrations with $\left(\sum \mathscr{E}_{X}\right) \cdot F=2 g-2$, $2 g-3$ whose total spaces are neither the blow-up of a rational surface nor the blow-up of a ruled surface. In fact, for such hyperelliptic genus- $g$ Lefschetz fibration $f: X \rightarrow S^{2}$, the number $\mu(f)$ of singular fibers of $f$ satisfies

$$
\mu(f) \leq \frac{(8 g-9)(2 g+1)}{g-1}+\sum_{h=1}^{[g / 2]} \frac{16 g^{2}-11 g-8}{12 h(g-h)-(2 g+1)} .
$$

Remark 6-3. (1) The estimation of $\mu(f)$ given in Corollary 6-2 is rough. By using linear programming, one can obtain a strict estimation of $\mu(f)$.

(2) There is no upper bound on the number of singular fibers of minimal Lefschetz fibrations. In fact, if a Lefschetz fibration $f: X \rightarrow S^{2}$ has $\mu$ singular fibers, then the fiber sum $m \#_{F} f$ of $m$ copies of $f$ is a minimal Lefschetz fibration with $m \mu$ singular fibers. Hence, there are minimal Lefschetz fibrations with arbitrarily many singular fibers.

Given $g(\geq 2)$, we can present the list of possible $([g / 2]+1)$-tuples

$$
\left(n_{0}, s_{1}, \ldots, s_{[g / 2]}\right)
$$

for nonminimal hyperelliptic genus- $g$ Lefschetz fibrations with $\left(\sum \mathscr{E}_{X}\right) \cdot F=2 g-2$, $2 g-3$. The lists in the cases of $g=2$ and $g=3$ are given in Tables 3 and 4 .

\begin{tabular}{|ccccc|}
\hline$\left(\sum \mathscr{E}_{X}\right) \cdot F$ & $\mathscr{E}_{X}$ & $K_{X}$ & $\left(n_{0}, s\right)$ & $\kappa^{s}$ \\
\hline \multirow{2}{*}{ Type $(1,1)$} & $K_{X}=E_{1}+E_{2}$ & $(16,2),(30,0)$ & \\
$\left(\sum \mathscr{E}_{X}\right) \cdot F=2$ & Type $(2)$ & $K_{X}=E$ & $(14,3),(28,1)$ & $\kappa^{s}=0$ \\
& & $K_{X}=2 E+R$ & $(28,1)$ & $\kappa^{s}=1$ \\
$\left(\sum \mathscr{E}_{X}\right) \cdot F=1$ & Type (1) & $K_{X}=2 E+F$ & $(40,0)$ & $\kappa^{s}=2$ \\
\hline
\end{tabular}

Table 3. Possible pairs $\left(n_{0}, s\right)$ as geography in the case of $g=2$. 


\begin{tabular}{|ccccc|}
\hline$\left(\sum \mathscr{E}_{X}\right) \cdot F$ & $\mathscr{E}_{X}$ & $K_{X}$ & $\left(n_{0}, s\right)$ & $\kappa^{s}$ \\
\hline & Type $(1,1,1,1)$ & $K_{X}=E_{1}+E_{2}+E_{3}+E_{4}$ & $(8,4)$ & \\
& Type $(1,1,2)$ & $K_{X}=E_{1}+E_{2}+E$ & $(20,3)$ & \\
$\left(\sum \mathscr{E}_{X}\right) \cdot F=4$ & Type $(1,3)$ & $K_{X}=E_{1}+E$ & $(32,2)$ & $\kappa^{s}=0$ \\
& Type $(2,2)$ & $K_{X}=E_{1}+E_{2}$ & $(32,2)$ & \\
& Type (4) & $K_{X}=E$ & $(44,1)$ & \\
\hline & Type $(1,1,1)$ & $K_{X}=2 E_{1}+E_{2}+E_{3}+R$ & $(20,3)$ & $\kappa^{s}=1$ \\
$\left(\sum \mathscr{E}_{X}\right) \cdot F=3$ & Type $(1,2)$ & $K_{X}=2 E_{1}+E_{2}+R$ & $(32,2)$ & $\kappa^{s}=1$ \\
& Type (3) & none & none & none \\
\hline
\end{tabular}

Table 4. Possible pairs $\left(n_{0}, s\right)$ as geography in the hyperelliptic case of $g=3$.

\section{The geography of nonminimal, nonhyperelliptic genus-3 Lefschetz fibrations.} At present, we do not know whether the signature $\sigma(X)$ of $X$ can be calculated from the number of singular fibers for a nonhyperelliptic Lefschetz fibration $f: X \rightarrow S^{2}$. Hence, we do not know whether the finiteness theorem of the geography holds for nonhyperelliptic case. However, in the case of nonhyperelliptic genus-3 Lefschetz fibrations, we can show the finiteness of the geography for nonminimal holomorphic Lefschetz fibrations by using Smith's signature formula and the Deligne-Mumford compactified moduli space $\bar{M}_{3}$ of stable curves of genus 3 .

Let $\triangle_{0}$ and $\triangle_{1}$ be the divisors of irreducible and reducible nodal curves, respectively. Then, the Deligne-Mumford compactified moduli space $\bar{M}_{3}$ is given by adjoining $\triangle_{0}$ and $\Delta_{1}$ to the moduli space $\mu_{3}$ of stable curves of genus 3 . Let $\overline{\mathscr{H}}_{3}$ denote the divisor of hyperelliptic curves of genus 3 in $\bar{M}_{3}$. Then, a theorem of Harer [1983] states that the Hodge class $c_{1}(\lambda),\left[\triangle_{0}\right]$ and $\left[\triangle_{1}\right]$ generate $H^{2}\left(\bar{M}_{3} ; \mathbb{Z}\right)$ and the cohomology class $\left[\overline{\mathscr{H}}_{3}\right]$ is given, up to a positive rational multiple, by

$$
\left[\overline{\mathscr{H}}_{3}\right]=9 c_{1}(\lambda)-\left[\triangle_{0}\right]-3\left[\triangle_{1}\right] \text {. }
$$

See [Harris and Morrison 1998].

Theorem 6-4. Suppose that $X$ is neither the blow-up of a rational surface nor the blow-up of a ruled surface. Then, only finitely many $\left(n_{0}, s\right)$ can be realized as pairs of the numbers of singular fibers for nonminimal, nonhyperelliptic and holomorphic genus-3 Lefschetz fibrations with $\left(\sum_{\mathscr{E}_{X}}^{\mathscr{E}_{X}}\right) \cdot F=3,4$.

Proof. Suppose that $f: X \rightarrow \mathbb{C P}^{1}$ is nonhyperelliptic and holomorphic. A holomorphic fibration $f$ gives rise to a rational curve $\phi_{f}\left(\mathbb{C P}^{1}\right)$ in $\bar{M}_{3}$. The rational curve $\phi_{f}\left(\mathbb{C P}^{1}\right)$ has positive intersection with any effective divisors that are not contained 
in $\phi_{f}\left(\mathbb{C P}^{1}\right)$. In particular, we have

$$
\left\langle\left[\overline{\mathscr{H}}_{3}\right],\left[\phi_{f}\left(\mathbb{C P}^{1}\right)\right]\right\rangle \geq 0 .
$$

Since $\left\langle\left[\overline{\mathscr{H}}_{3}\right],\left[\phi_{f}\left(\mathbb{C P}^{1}\right)\right]\right\rangle$ is given, up to a positive multiple, by

$$
\begin{aligned}
\left\langle\left[\overline{\mathscr{H}}_{3}\right],\left[\phi_{f}\left(\mathbb{C} P^{1}\right)\right]\right\rangle & =\left\langle 9 c_{1}(\lambda)-\left[\triangle_{0}\right]-3\left[\triangle_{1}\right],\left[\phi_{f}\left(\mathbb{C} P^{1}\right)\right]\right\rangle \\
& =\frac{9}{4}\left(\sigma(X)+n_{0}+s\right)-n_{0}-3 s \\
& =\frac{9}{4} \sigma(X)+\frac{5}{4} n_{0}-\frac{3}{4} s,
\end{aligned}
$$

we can obtain the following inequality:

$$
\sigma(X) \geq-\frac{5}{9} n_{0}+\frac{1}{3} s .
$$

Thus, we get the relations

$$
\left\{\begin{array}{l}
K_{X}^{2}=3 \sigma(X)+2 e(X) \\
-4 \leq K_{X}^{2} \leq-1, \\
\sigma(X) \geq-\frac{5}{9} n_{0}+\frac{1}{3} s, \\
e(X)=n_{0}+s-8, \\
5 n_{0} \geq s,
\end{array}\right.
$$

hence

$$
\left\{\begin{array}{l}
n_{0}+9 s-45 \leq 0 \\
5 n_{0}-s \geq 0 \\
n_{0}>0, s \geq 0
\end{array}\right.
$$

The number of pairs $\left(n_{0}, s\right)$ satisfying these inequalities is finite.

\section{Acknowledgments}

The author would like to thank Professors Tadashi Ashikaga, Hisaaki Endo and Susumu Hirose for helpful discussions. The author is also very grateful to the referee for his comments and suggestions.

\section{References}

[Auroux 2003] D. Auroux, "Fiber sums of genus 2 Lefschetz fibrations", Turkish J. Math. 27:1 (2003), 1-10. MR 2004b:57033 Zbl 1075.53087

[Auroux 2006a] D. Auroux, "The canonical pencils on Horikawa surfaces”, Geom. Topol. 10 (2006), 2173-2217. MR 2007m:14065 Zbl 1129.57030

[Auroux 2006b] D. Auroux, "Mapping class group factorizations and symplectic 4-manifolds: some open problems", pp. 123-132 in Problems on mapping class groups and related topics, edited by B. Farb, Proc. Sympos. Pure Math. 74, Amer. Math. Soc., Providence, RI, 2006. MR 2007h:53134 Zbl 05124680 
[Donaldson 1987] S. K. Donaldson, "Irrationality and the $h$-cobordism conjecture", J. Differential Geom. 26:1 (1987), 141-168. MR 88j:57035 Zbl 0631.57010

[Donaldson 1998] S. K. Donaldson, "Lefschetz fibrations in symplectic geometry", Doc. Math. Extra Vol. 2 (1998), 309-314. MR 99i:57044 Zbl 0909.53018

[Dorfmeister and Zhang 2009] J. Dorfmeister and W. Zhang, "The Kodaira dimension of Lefschetz fibrations”, Asian J. Math. 13:3 (2009), 341-357. MR 2010k:53147 Zbl 1207.53086

[Endo 2000] H. Endo, "Meyer's signature cocycle and hyperelliptic fibrations", Math. Ann. 316:2 (2000), 237-257. MR 2001b:57047 Zbl 0948.57013

[Endo 2008] H. Endo, "A generalization of Chakiris' fibrations", pp. 251-282 in Groups of diffeomorphisms, edited by R. Penner et al., Adv. Stud. Pure Math. 52, Math. Soc. Japan, Tokyo, 2008. MR 2011e:57044 Zbl 1180.57028

[Fintushel and Stern 1998] R. Fintushel and R. J. Stern, "Constructions of smooth 4-manifolds", Doc. Math. Extra Vol. 2 (1998), 443-452. MR 99g:57033 Zbl 0899.57012

[Fuller 1998] T. Fuller, "Diffeomorphism types of genus 2 Lefschetz fibrations", Math. Ann. 311:1 (1998), 163-176. MR 99f:57035 Zbl 0905.57014

[Gompf 1995] R. E. Gompf, "A new construction of symplectic manifolds", Ann. of Math. (2) 142:3 (1995), 527-595. MR 96j:57025 Zbl 0849.53027

[Gompf and Stipsicz 1999] R. E. Gompf and A. I. Stipsicz, 4-manifolds and Kirby calculus, Graduate Studies in Mathematics 20, American Mathematical Society, Providence, RI, 1999. MR 2000h:57038 Zbl 0933.57020

[Harer 1983] J. Harer, "The second homology group of the mapping class group of an orientable surface”, Invent. Math. 72:2 (1983), 221-239. MR 84g:57006 Zbl 0533.57003

[Harris and Morrison 1998] J. Harris and I. Morrison, Moduli of curves, Graduate Texts in Mathematics 187, Springer, New York, 1998. MR 99g:14031 Zbl 0913.14005

[Kametani and Sato 1994] Y. Kametani and Y. Sato, "0-dimensional moduli space of stable rank 2 bundles and differentiable structures on regular elliptic surfaces", Tokyo J. Math. 17:1 (1994), 253-267. MR 95g:14012 Zbl 0826.14023

[Korkmaz and Ozbagci 2001] M. Korkmaz and B. Ozbagci, "Minimal number of singular fibers in a Lefschetz fibration", Proc. Amer. Math. Soc. 129:5 (2001), 1545-1549. MR 2001h:57019 Zbl 1058.57011

[Kuno 2010] Y. Kuno, "On the global monodromy of a Lefschetz fibration arising from the Fermat surface of degree 4”, Kodai Math. J. 33:3 (2010), 457-472. MR 2754332 Zbl 1221.14011

[Li 1999] T.-J. Li, "Smoothly embedded spheres in symplectic 4-manifolds", Proc. Amer. Math. Soc. 127:2 (1999), 609-613. MR 99c:57055 Zbl 0911.57018

[Li 2006] T.-J. Li, "Symplectic 4-manifolds with Kodaira dimension zero", J. Differential Geom. 74:2 (2006), 321-352. MR 2007e:53114 Zbl 1105.53068

[Li and Liu 1995] T. J. Li and A. Liu, "Symplectic structure on ruled surfaces and a generalized adjunction formula", Math. Res. Lett. 2:4 (1995), 453-471. MR 96m:57052 Zbl 0855.53019

[Liu 1996] A.-K. Liu, "Some new applications of general wall crossing formula, Gompf's conjecture and its applications", Math. Res. Lett. 3:5 (1996), 569-585. MR 97k:57038 Zbl 0872.57025

[Matsumoto 1986] Y. Matsumoto, "Diffeomorphism types of elliptic surfaces", Topology 25:4 (1986), 549-563. MR 88b:32061 Zbl 0615.14023

[Matsumoto 1996] Y. Matsumoto, "Lefschetz fibrations of genus two-a topological approach", pp. 123-148 in Topology and Teichmüller spaces (Katinkulta, 1995), edited by S. Kojima et al., World Scientific, River Edge, NJ, 1996. MR 2000h:14038 Zbl 0921.57006 
[McCarthy and Wolfson 1994] J. D. McCarthy and J. G. Wolfson, "Symplectic normal connect sum", Topology 33:4 (1994), 729-764. MR 95h:57038 Zbl 0812.53033

[McDuff and Salamon 1996] D. McDuff and D. Salamon, "A survey of symplectic 4-manifolds with $b^{+}=1$ ", Turkish J. Math. 20:1 (1996), 47-60. MR 97e:57028 Zbl 0870.57023

[Meyer 1973] W. Meyer, "Die Signatur von Flächenbündeln”, Math. Ann. 201 (1973), 239-264. MR 48 \#9715 Zbl 0241.55019

[Ohta and Ono 2005] H. Ohta and K. Ono, "Symplectic 4-manifolds containing singular rational curves with (2, 3)-cusp", pp. 233-241 in Singularités Franco-Japonaises, edited by J.-P. Brasselet and T. Suwa, Sémin. Congr. 10, Soc. Math. France, Paris, 2005. MR 2006g:53140 Zbl 1082.53080

[Ozbagci and Stipsicz 2000] B. Ozbagci and A. I. Stipsicz, "Noncomplex smooth 4-manifolds with genus-2 Lefschetz fibrations", Proc. Amer. Math. Soc. 128:10 (2000), 3125-3128. MR 2000m:57036 Zbl 0951.57015

[Persson 1987] U. Persson, "An introduction to the geography of surfaces of general type", pp. 195218 in Algebraic geometry, Bowdoin, 1985 (Brunswick, Maine, 1985), edited by S. J. Bloch, Proc. Sympos. Pure Math. 46, Amer. Math. Soc., Providence, RI, 1987. MR 89a:14057 Zbl 0656.14020

[Persson 1992] U. Persson, "Genus two fibrations revisited", pp. 133-144 in Complex algebraic varieties (Bayreuth, 1990), edited by K. Hulek et al., Lecture Notes in Math. 1507, Springer, Berlin, 1992. MR 1178724 Zbl 0792.14018

[Sato 2006] Y. Sato, “The Stipsicz's conjecture for genus-2 Lefschetz fibrations”, preprint, 2006.

[Sato 2008] Y. Sato, "2-spheres of square -1 and the geography of genus-2 Lefschetz fibrations", $J$. Math. Sci. Univ. Tokyo 15:4 (2008), 461-491. MR 2011c:57059 Zbl 1236.57036

[Sato 2010a] Y. Sato, "An attempt to introduce the notion of the Iitaka-Kodaira dimension into Lefschetz fibrations", preprint, 2010.

[Sato 2010b] Y. Sato, "The necessary condition on the fiber-sum decomposability of genus-2 Lefschetz fibrations”, Osaka J. Math. 47:4 (2010), 949-963. MR 2012j:57052 Zbl 1228.57011

[Smith 1999] I. Smith, "Lefschetz fibrations and the Hodge bundle", Geom. Topol. 3 (1999), 211-233. MR 2000j:57059 Zbl 0929.53047

[Smith 2001a] I. Smith, "Geometric monodromy and the hyperbolic disc", Q. J. Math. 52:2 (2001), 217-228. MR 2002c:57046 Zbl 0981.57013

[Smith 2001b] I. Smith, "Lefschetz pencils and divisors in moduli space", Geom. Topol. 5 (2001), 579-608. MR 2002f:57056 Zbl 1066.57030

[Smith 2001c] I. Smith, "Torus fibrations on symplectic four-manifolds", Turkish J. Math. 25:1 (2001), 69-95. MR 2002c:57047 Zbl 0996.53055

[Stipsicz 1996] A. Stipsicz, "A note on the geography of symplectic manifolds", Turkish J. Math. 20:1 (1996), 135-139. MR 97m:57035 Zbl 0876.57039

[Stipsicz 1998] A. I. Stipsicz, "Simply connected 4-manifolds near the Bogomolov-Miyaoka-Yau line”, Math. Res. Lett. 5:6 (1998), 723-730. MR 2000h:57047 Zbl 0947.57032

[Stipsicz 1999] A. I. Stipsicz, "On the number of vanishing cycles in Lefschetz fibrations", Math. Res. Lett. 6:3-4 (1999), 449-456. MR 2000g:57046 Zbl 0955.57026

[Stipsicz 2000] A. I. Stipsicz, "The geography problem of 4-manifolds with various structures", Acta Math. Hungar. 87:4 (2000), 267-278. MR 2001g:57050 Zbl 0958.53034

[Stipsicz 2001] A. I. Stipsicz, "Indecomposability of certain Lefschetz fibrations", Proc. Amer. Math. Soc. 129:5 (2001), 1499-1502. MR 2001h:57029 Zbl 0978.57022

[Taubes 1994] C. H. Taubes, "The Seiberg-Witten invariants and symplectic forms", Math. Res. Lett. 1:6 (1994), 809-822. MR 95j:57039 Zbl 0853.57019 
[Taubes 1995] C. H. Taubes, “The Seiberg-Witten and Gromov invariants", Math. Res. Lett. 2:2 (1995), 221-238. MR 96a:57076 Zbl 0854.57020

[Taubes 1996] C. H. Taubes, "SW $\Rightarrow$ Gr: From the Seiberg-Witten equations to pseudo-holomorphic curves”, J. Amer. Math. Soc. 9:3 (1996), 845-918. MR 97a:57033 Zbl 0867.53025

[Thurston 1976] W. P. Thurston, "Some simple examples of symplectic manifolds", Proc. Amer. Math. Soc. 55:2 (1976), 467-468. MR 53 \#6578 Zbl 0324.53031

[Ue 1986] M. Ue, "On the diffeomorphism types of elliptic surfaces with multiple fibers", Invent. Math. 84:3 (1986), 633-643. MR 87j:57019 Zbl 0595.14028

[Usher 2006] M. Usher, "Minimality and symplectic sums", Int. Math. Res. Not. 2006:16 (2006), 1-17. Art. ID 49857. MR 2007h:53139 Zbl 1110.57017

Received August 3, 2011. Revised July 11, 2012.

YOSHIHISA SATO

DEPARTMENT OF SYSTEMS DESIGN AND INFORMATICS

KYUSHU INSTITUTE OF TECHNOLOGY

680-4 KAWAZU, IIZUKA

FUKUOKA 820-8502

JAPAN

ysato@ces.kyutech.ac.jp 


\title{
PACIFIC JOURNAL OF MATHEMATICS
}

\author{
msp.org/pjm
}

Founded in 1951 by E. F. Beckenbach (1906-1982) and F. Wolf (1904-1989)

\section{EDITORS}

V. S. Varadarajan (Managing Editor)

Department of Mathematics

University of California

Los Angeles, CA 90095-1555

pacific@math.ucla.edu

Paul Balmer

Department of Mathematics

University of California

Los Angeles, CA 90095-1555

balmer@math.ucla.edu

Daryl Cooper

Department of Mathematics

University of California

Santa Barbara, CA 93106-3080 cooper@math.ucsb.edu

Jiang-Hua $\mathrm{Lu}$

Department of Mathematics

The University of Hong Kong

Pokfulam Rd., Hong Kong jhlu@maths.hku.hk
Don Blasius

Department of Mathematics University of California

Los Angeles, CA 90095-1555

blasius@math.ucla.edu

Robert Finn

Department of Mathematics Stanford University

Stanford, CA 94305-2125

finn@math.stanford.edu

Sorin Popa

Department of Mathematics

University of California

Los Angeles, CA 90095-1555

popa@math.ucla.edu

Paul Yang

Department of Mathematics

Princeton University

Princeton NJ 08544-1000

yang@math.princeton.edu

\section{PRODUCTION}

Silvio Levy, Scientific Editor, production@msp.org

\section{SUPPORTING INSTITUTIONS}

ACADEMIA SINICA, TAIPEI

CALIFORNIA INST. OF TECHNOLOGY

INST. DE MATEMÁTICA PURA E APLICADA

KEIO UNIVERSITY

MATH. SCIENCES RESEARCH INSTITUTE

NEW MEXICO STATE UNIV.

OREGON STATE UNIV.

\author{
STANFORD UNIVERSITY \\ UNIV. OF BRITISH COLUMBIA \\ UNIV. OF CALIFORNIA, BERKELEY \\ UNIV. OF CALIFORNIA, DAVIS \\ UNIV. OF CALIFORNIA, LOS ANGELES \\ UNIV. OF CALIFORNIA, RIVERSIDE \\ UNIV. OF CALIFORNIA, SAN DIEGO \\ UNIV. OF CALIF., SANTA BARBARA
}

\author{
Vyjayanthi Chari \\ Department of Mathematics \\ University of California \\ Riverside, CA 92521-0135 \\ chari@math.ucr.edu \\ Kefeng Liu \\ Department of Mathematics \\ University of California \\ Los Angeles, CA 90095-1555 \\ liu@math.ucla.edu \\ Jie Qing \\ Department of Mathematics \\ University of California \\ Santa Cruz, CA 95064 \\ qing@cats.ucsc.edu
}

These supporting institutions contribute to the cost of publication of this Journal, but they are not owners or publishers and have no responsibility for its contents or policies.

See inside back cover or msp.org/pjm for submission instructions.

The subscription price for 2013 is US \$400/year for the electronic version, and \$485/year for print and electronic.

Subscriptions, requests for back issues and changes of subscribers address should be sent to Pacific Journal of Mathematics, P.O. Box 4163, Berkeley, CA 94704-0163, U.S.A. The Pacific Journal of Mathematics is indexed by Mathematical Reviews, Zentralblatt MATH, PASCAL CNRS Index, Referativnyi Zhurnal, Current Mathematical Publications and the Science Citation Index.

The Pacific Journal of Mathematics (ISSN 0030-8730) at the University of California, c/o Department of Mathematics, 798 Evans Hall \#3840, Berkeley, CA 94720-3840, is published monthly except July and August. Periodical rate postage paid at Berkeley, CA 94704, and additional mailing offices. POSTMASTER: send address changes to Pacific Journal of Mathematics, P.O. Box 4163, Berkeley, CA 94704-0163.

PJM peer review and production are managed by EditFLOW ${ }^{\circledR}$ from Mathematical Sciences Publishers.

PUBLISHED BY

mathematical sciences publishers

nonprofit scientific publishing

http://msp.org/

(C) 2013 Mathematical Sciences Publishers 


\section{PACIFIC JOURNAL OF MATHEMATICS}

Volume $262 \quad$ No. $1 \quad$ March 2013

On the second $K$-group of a rational function field

KARIM JOHANNES BECHER and MÉLANIE RACZEK

On existence of a classical solution to a generalized Kelvin-Voigt model

Miroslav BulíčEK, Petr KaPliCKÝ and Mark

STEINHAUER

A lower bound for eigenvalues of the poly-Laplacian with arbitrary order

Qing-Ming Cheng, Xuerong Qi and GuOXIN Wei

Quiver algebras, path coalgebras and coreflexivity

SORIN DĂSCĂLESCU, MIODRAG C. IOVANOV and CONSTANTIN

NĂSTĂSESCU

A positive density of fundamental discriminants with large regulator

ÉTIENNE FOUVRY and FLORENT JOUVE

On the isentropic compressible Euler equation with adiabatic index $\gamma=1$

Dong Li, ChANGXing MiaO and XiaOyi Zhang

Symmetric regularization, reduction and blow-up of the planar three-body problem

RICHARD MOECKEL and RICHARD MONTGOMERY

Canonical classes and the geography of nonminimal Lefschetz fibrations over $S^{2}$

YOSHIHISA SATO

Hilbert-Kunz invariants and Euler characteristic polynomials 